\title{
Elliptic ovoids and their rosettes in a classical generalized quadrangle of even order
}

\author{
ILARIA CARDINALI ${ }^{1, *}$ and N S NARASIMHA SASTRY ${ }^{2}$ \\ ${ }^{1}$ Department of Information Engineering and Mathematics, University of Siena, \\ Via Roma 56, I-53100, Siena, Italy \\ ${ }^{2}$ Theoretical Statistics and Mathematics Unit, Indian Statistical Institute, \\ 8th Mile Mysore Road, Bangalore 560 059, India \\ * Corresponding author. \\ E-mail: ilaria.cardinali@unisi.it; nsastry@ isibang.ac.in
}

MS received 22 October 2014; revised 19 October 2015

\begin{abstract}
Let $Q_{0}$ be the classical generalized quadrangle of order $q=2^{n}(n \geq 2)$ arising from a non-degenerate quadratic form in a 5-dimensional vector space defined over a finite field of order $q$. We consider the rank two geometry $\mathcal{X}$ having as points all the elliptic ovoids of $Q_{0}$ and as lines the maximal pencils of elliptic ovoids of $Q_{0}$ pairwise tangent at the same point. We first prove that $\mathcal{X}$ is isomorphic to a 2-fold quotient of the affine generalized quadrangle $Q \backslash Q_{0}$, where $Q$ is the classical $\left(q, q^{2}\right)$ generalized quadrangle admitting $Q_{0}$ as a hyperplane. Further, we classify the cliques in the collinearity graph $\Gamma$ of $\mathcal{X}$. We prove that any maximal clique in $\Gamma$ is either a line of $\mathcal{X}$ or it consists of 6 or 4 points of $\mathcal{X}$ not contained in any line of $\mathcal{X}$, accordingly as $n$ is odd or even. We count the number of cliques of each type and show that those cliques which are not contained in lines of $\mathcal{X}$ arise as subgeometries of $Q$ defined over $\mathbb{F}_{2}$.
\end{abstract}

Keywords. Generalized quadrangles; collinearity graph; $t$-fold covering; cliques.

2010 Mathematics Subject Classification. 51E12, 05B25, $05 \mathrm{C} 69$.

\section{Introduction}

We refer to [10] for the terminology and basics on finite generalized quadrangles. We only recall that a finite generalized quadrangle $\mathcal{Q}$ is classical if its points and lines are points and lines of a projective space $\operatorname{PG}(d, q)$, the points of $\mathcal{Q}$ span $\operatorname{PG}(d, q)$ and if a projective line $l$ is a line of $\mathcal{Q}$, then all points of $l$ belong to $\mathcal{Q}$. If this is the case, then the points and the lines of $\mathcal{Q}$ are the points and the lines of $\operatorname{PG}(d, q)$ that are singular and totally singular, respectively, for a suitable non-degenerate alternating, hermitian or quadratic form of the underlying vector space $V(d+1, q)$ of $\operatorname{PG}(d, q)$ (Chapter 4 of [10]).

The generalized quadrangle of order $q$ arising from a non-degenerate symplectic form on $V(4, q)$ is denoted by $W(q)$. The generalized quadrangle of order $q$ arising from a non-degenerate quadratic form in $V(5, q)$ is denoted by $Q(4, q)$. The $\left(q, q^{2}\right)$-generalized quadrangle arising from a non-degenerate quadratic form of Witt index 2 in $V(6, q)$ is denoted by $Q^{-}(5, q)$. The $(q, 1)$-generalized quadrangle arising from a non-degenerate quadratic form of Witt index 2 in $V(4, q)$ is denoted by $Q^{+}(3, q)$. It is also called a grid. 
For any $q$, the dual of $Q(4, q)$ is isomorphic to $W(q)$. Moreover, $Q(4, q) \cong W(q)$ if and only if $q$ is even.

An ovoid of an $(s, t)$-generalized quadrangle $\mathcal{Q}$ is a set $X$ of points of $\mathcal{Q}$ such that every line of $\mathcal{Q}$ meets $X$ at exactly one point (see [10]). A simple count shows that $|X|=s t+1$. By definition, no two points of $X$ are collinear. For a survey of ovoids of generalized quadrangles, we refer the reader to [12].

In this paper, we assume that $q=2^{n}$ and denote by $Q_{0}$ a classical generalized quadrangle $Q(4, q)$ of order $q$ embedded in a 4-dimensional projective space $\mathrm{PG}\left(V_{0}\right), V_{0}=$ $V(5, q)$. Let $n_{0}$ be the nucleus of $Q_{0}$, i.e., $n_{0}$ is the unique non-singular point of $\mathrm{PG}\left(V_{0}\right)$ with the property that the lines of $\mathrm{PG}\left(V_{0}\right)$ through $n_{0}$ are the tangents to $Q_{0}$ in $\mathrm{PG}\left(V_{0}\right)$ (see, e.g., Chapter 22.3 of [6]).

Classifying ovoids in $Q_{0}$ is a fundamental open problem. There are two known classes of ovoids of $Q_{0}$, namely the class $\mathcal{E}$ of elliptic ovoids and the class $\mathcal{T}$ of Tits-Suzuki ovoids. Tits-Suzuki ovoids exist if and only if $n$ is odd. An ovoid $X$ of $Q_{0}$ is elliptic if and only if it spans a hyperplane of $\operatorname{PG}\left(V_{0}\right)$ (whence $X=\langle X\rangle \cap Q_{0}$ ).

We recall that any two distinct elliptic ovoids of $Q_{0}$ intersect in either a singleton or a non-degenerate conic. If two elliptic ovoids meet at a single point $p$, we say that they are tangent at $p$.

Following [2], we define a rosette $\rho_{p}$ of elliptic ovoids based at a point $p$ as a set of $q$ elliptic ovoids of $Q_{0}$ mutually tangent at $p$ such that $\left\{X \backslash\{p\}: X \in \rho_{p}\right\}$ is a partition of the set of the points of $Q_{0}$ not collinear with $p$. The point $p$ is called the base point of $\rho_{p}$. Clearly, a set of elliptic ovoids mutually tangent at a given point is a rosette if and only if it consists of exactly $q$ ovoids. It is also clear that a rosette with base point $p$ is a maximal pencil of elliptic ovoids of $Q_{0}$ mutually tangent at $p$.

The next Proposition summarizes some properties of elliptic ovoids and rosettes of $Q_{0}$. Proof of Proposition 1.1(a) and (b) can be found in Theorem 3.2 of [1] (see also Example 1.4(d) of [5]). Parts (c) and (d) of Proposition 1.1 are proved in $\S 3$.

\section{PROPOSITION 1.1}

\section{The following hold:}

(a) Let $X_{1}$ and $X_{2}$ be two elliptic ovoids of $Q_{0}$ tangent at a point $p$. Then there exists a unique rosette $\rho_{p}$ with base point $p$ containing $X_{1}$ and $X_{2}$.

(b) Two elliptic ovoids of $Q_{0}$ in distinct rosettes based at the same point $p$ meet in a conic containing $p$.

(c) Let $X_{1}$ and $X_{2}$ be two elliptic ovoids of $Q_{0}$ tangent at a point. For each $x \in X_{1} \backslash X_{2}$, there exists a unique elliptic ovoid of $Q_{0}$ through $x$ which is tangent to both $X_{1}$ and $X_{2}$.

(d) Let $X_{1}$ and $X_{2}$ be two elliptic ovoids of $Q_{0}$ intersecting in a conic. If $x \in X_{1} \backslash X_{2}$ then there exist exactly two distinct elliptic ovoids of $Q_{0}$ through $x$ which are both tangent to $X_{1}$ and $X_{2}$. If $x \in X_{1} \cap X_{2}$ then there exist no elliptic ovoids of $Q_{0}$ through $x$ which are both tangent to $X_{1}$ and $X_{2}$.

\section{PROPOSITION 1.2}

Let $\rho_{p}$ be a rosette of elliptic ovoids of $Q_{0}$ based at a point $p$. Then there exists a unique projective plane $\pi_{p}$ of $\mathrm{PG}\left(V_{0}\right)$ containing $p$ and tangent to each $X \in \rho_{p}$. 
Proof. Let $X_{i}, X_{j}, X_{k}$ be three distinct elliptic ovoids in $\rho_{p}$. Let $\pi_{j}:=\left\langle X_{i}\right\rangle \cap\left\langle X_{j}\right\rangle$ and $\pi_{k}:=\left\langle X_{i}\right\rangle \cap\left\langle X_{k}\right\rangle$ be the planes of the 3-dimensional subspace $\left\langle X_{i}\right\rangle$ spanned by $X_{i}$ obtained as its intersection with the 3-dimensional subspaces spanned by $X_{j}$ and $X_{k}$, respectively. Since $\langle X\rangle \cap Q_{0}=X$, for every $X \in \mathcal{E}$, we have $\pi_{j} \cap X_{i}=p=$ $\pi_{k} \cap X_{i}$

If $\pi_{j} \neq \pi_{k}$, then $\pi_{j}$ and $\pi_{k}$ are two distinct planes of $\left\langle X_{i}\right\rangle$ both tangent to $X_{i}$ at $p$. This is clearly impossible. Hence, $\pi_{j}=\pi_{k}$ and the proposition follows.

Let $\mathcal{X}:=(\mathcal{E}, \mathcal{L})$ be the point-line geometry having the set $\mathcal{E}$ of elliptic ovoids of $Q_{0}$ as the point set and the set of rosettes of elliptic ovoids of $Q_{0}$ as the line set. An elliptic ovoid $X$ is incident with a rosette $\rho_{p}$ if $X \in \rho_{p}$. The geometry $\mathcal{X}$ is a semi-partial geometry with parameters $(s, t, \alpha, \mu)=\left(q-1, q^{2}, 2,2 q(q-1)\right)$ (see [5] and [4]). A very straightforward proof of this claim will also be given in $\S 2$.

\subsection{Main results}

Let $Q \cong Q^{-}(5, q)$ be a classical $\left(q, q^{2}\right)$-generalized quadrangle embedded in the 5-dimensional projective space $\operatorname{PG}(V)$, where $V=V(6, q)$. We may assume that $Q_{0}=$ $Q \cap H_{0}$ for a suitable hyperplane $H_{0}$ of $\mathrm{PG}(V)$. Thus, $Q_{0}$ is embedded in $H_{0} \cong \mathrm{PG}(4, q)$. Let $\perp$ denote the orthogonality relation defined by $Q$ in $\operatorname{PG}(V)$. The point $n_{0}:=H_{0}^{\perp}$ is the nucleus of the quadric $Q_{0}$.

Let $\hat{\mathcal{X}}:=(\hat{P}, \hat{L})$ be the point-line geometry having the set $\hat{P}$ of the points of $Q \backslash Q_{0}$ as the point set and the set $\hat{L}:=\left\{l \backslash Q_{0}: l\right.$ a line of $Q$ not contained in $\left.Q_{0}\right\}$ as the line set. This geometry is called affine generalized quadrangle.

If $l$ is a line of $Q$ not contained in $Q_{0}$, then $l \cap Q_{0}$ is a point, henceforth denoted by $l^{\infty}$. We denote by $\hat{l}$ the line of $\hat{\mathcal{X}}$ corresponding to $l$ and we call $l^{\infty}$ the point at infinity of $\hat{\imath}$. Thus,

$$
\hat{l}=l \backslash\left\{l^{\infty}\right\} \quad \text { and } \quad l=\hat{l} \cup\left\{l^{\infty}\right\}
$$

The incidence relation in $\hat{\mathcal{X}}$ is inherited from the incidence relation of $Q$. It is easy to see that the incidence graph of $\hat{\mathcal{X}}$ is connected. It has girth 8 , whence $\hat{\mathcal{X}}$ has gonality 4 . The collinearity graph $\hat{\Gamma}$ of $\hat{\mathcal{X}}$ is regular and has diameter 3 . It contains 4-circuits and every clique of $\hat{\Gamma}$ is contained in a line of $\hat{\mathcal{X}}$ (see, e.g., $\$ 8.4 .1$ of [9]).

We recall that a morphism of rank 2 geometries is a morphism of their incidence graphs (see, e.g., Chapter 8 of [9]). Given two rank 2 geometries $\mathcal{G}_{1}$ and $\mathcal{G}_{2}$, a morphism $\varphi: \mathcal{G}_{1} \rightarrow$ $\mathcal{G}_{2}$ is a covering from $\mathcal{G}_{1}$ to $\mathcal{G}_{2}$ if, for any point $p$ of $\mathcal{G}_{1}$ and any line $l$ of $\mathcal{G}_{1}, \varphi$ induces a bijection from the point set of $l$ to the point set of $\varphi(l)$ and from the set of lines of $\mathcal{G}_{1}$ incident with $p$ to the set of lines of $\mathcal{G}_{2}$ incident with $\varphi(p)$. If each fiber of the covering has size $t$, then we say that $\varphi$ is a $t$-fold covering of $\mathcal{G}_{2}, \mathcal{G}_{1}$ is called a $t$-fold cover of $\mathcal{G}_{2}$ and $\mathcal{G}_{2}$ is called a $t$-fold quotient of $\mathcal{G}_{1}$. Note that all coverings of connected geometries are surjective (see $\$ 8.3$ of [9]). In the terminology of graph theory, a covering of geometries is a covering of their incidence graphs. It also induces a covering of the collinearity graphs, satisfying the additional property that, if $a, b, c$ are different points of $\mathcal{G}_{1}$ with $b$ and $c$ collinear with $a$ and $\varphi(a), \varphi(b), \varphi(c)$ belonging to a common line of $\mathcal{G}_{2}$, then $a, b, c$ belong to the same line of $\mathcal{G}_{1}$. We warn that, however, if $b$ and $c$ are collinear with $a$ and, $\varphi(b)$ and $\varphi(c)$ are mutually collinear but not on the same line as $\varphi(a)$, then $b$ and $c$ need not be collinear in $\mathcal{G}_{1}$. 
In Section 2, we prove:

Theorem 1. The geometry $\hat{\mathcal{X}}$ is a 2-fold cover of the geometry $\mathcal{X}$. Explicitly, let $\varphi_{0}: \hat{\mathcal{X}} \rightarrow$ $\mathcal{X}$ map $x \in \hat{P}$ to $x^{\perp} \cap Q_{0}$ and $\hat{l} \in \hat{L}$ to $\rho_{p}$, where $p=l^{\infty}$. Then $\varphi_{0}$ is a 2-fold covering of $\mathcal{X}$.

The covering $\varphi_{0}$ defined above is called the canonical covering from $\hat{\mathcal{X}}$ to $\mathcal{X}$. As $Q$ can be recovered from $\hat{\mathcal{X}}$ (see Theorem 2 of [11]), every automorphism $\hat{\alpha}$ of $\hat{\mathcal{X}}$ can be extended to a unique automorphism $\alpha$ of $Q$ stabilizing $Q_{0}$. Let $\alpha_{0}$ be the automorphism of $Q_{0}$ induced by $\alpha$. Clearly, $\varphi_{0} \circ \hat{\alpha}=\alpha_{0} \circ \varphi_{0}$. It follows that the composition $\varphi:=\varphi_{0} \circ \hat{\alpha}$ is still a 2-fold covering of $\mathcal{X}$. Perhaps, all 2-fold coverings from $\hat{\mathcal{X}}$ to $\mathcal{X}$ arise in this way, but we will not pursue the conjecture in this paper.

In graph theory the classification and characterization of the maximal cliques of a graph is one of the most interesting and difficult problems to address. For the graph we are interested in, relying on the underlying geometry, where we succeed in both the classification and characterization. Indeed, in $\$ 3$, we determine the cliques of the collinearity graph $\Gamma$ of $\mathcal{X}$. A clique of $\Gamma$ is linear if it is a subset of a line of $\mathcal{X}$; non-linear otherwise. A clique of size $i$ is called an $i$-clique.

As we will prove in $\$ 3$ (Proposition 3.2), a linear clique and a non-linear clique meet in at most two vertices.

Theorem 2. Let $q=2^{n}>2$. The following hold:

(a) If $n$ is even, then a maximal clique of $\Gamma$ has either $q$ or 4 elements. Further, $q$-cliques are linear and maximal 4-cliques are non-linear.

(b) If $n$ is odd, then a maximal clique of $\Gamma$ has either $q$ or 6 elements. Further, $q$-cliques are linear and maximal 6-cliques are non-linear.

(c) Every non-linear 3-clique can be extended to $q+1$ distinct non-linear 4-cliques.

(d) If $n$ is odd, then

(i) every non-linear 4-clique extends precisely to two non-linear 5-cliques;

(ii) every non-linear 5-clique extends uniquely to a non-linear 6-clique.

We remark that $\Gamma$ is a complete graph on 6 vertices if $q=2$. If $q=4$, all maximal cliques have size 4 . Denote by $\hat{\Gamma}$ the collinearity graph of $\hat{\mathcal{X}}$.

A hexagon $H=\left(a^{1}, b^{1}, c^{1}, a^{2}, b^{2}, c^{2}, a^{1}\right)$ of $\hat{\mathcal{X}}$ is a 6-circuit of $\hat{\Gamma}$ (figure 1$)$. Note that $H$ admits a bipartition by two 3 -sets $\left\{a^{1}, b^{1}, c^{1}\right\}$ and $\left\{a^{2}, b^{2}, c^{2}\right\}$ as well as a partition by three 2 -sets $\left\{a^{1}, a^{2}\right\},\left\{b^{1}, b^{2}\right\}$ and $\left\{c^{1}, c^{2}\right\}$ such that vertices in the same 2-set are on a secant line to $Q$ through $n_{0}$. Vertices in the same 2 -set are called opposite. We say that a hexagon is centric if all projective lines through opposite vertices are concurrent. The point of concurrence is called the center of the hexagon.

A cube $C$ of $\hat{\mathcal{X}}$ is an induced subgraph of $\hat{\Gamma}$ with 8 vertices as in figure 2, where adjacents are represented by thick lines.

The graph $C$ admits a partition by two 4-sets $\left\{a^{1}, b^{1}, c^{1}, d^{1}\right\},\left\{a^{2}, b^{2}, c^{2}, d^{2}\right\}$ as well as a partition by four 2-sets $\left\{a^{1}, a^{2}\right\},\left\{b^{1}, b^{2}\right\},\left\{c^{1}, c^{2}\right\},\left\{d^{1}, d^{2}\right\}$, two vertices in a 2 -set being called opposite. We say that a cube is centric if all projective lines through opposite vertices are concurrent. The point of concurrence is called the center of the cube.

Note that a hexagon (respectively, a cube) is the complement of a $(3 \times 2)$-grid graph (respectively, a $(4 \times 2)$-grid graph). The bipartition in two classes of size 3 (respectively, 4 ) 


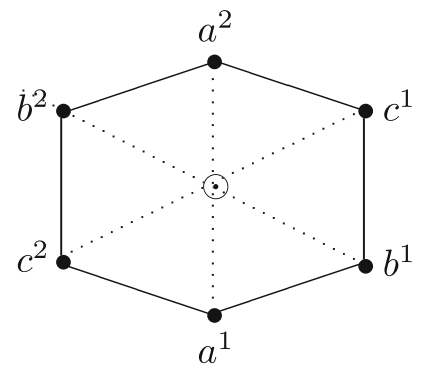

Figure 1. Centric hexagon.

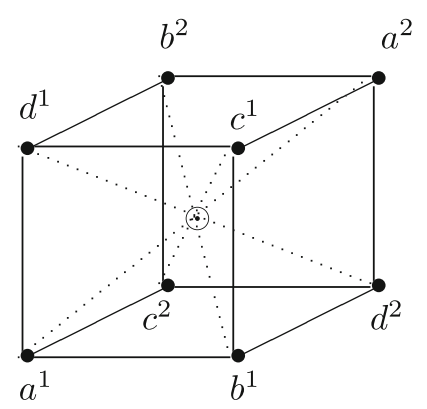

Figure 2. Centric cube.

is one of the two families of lines of the grid and the partition by three (respectively four) 2 -sets is the other family. We exploit this observation to define dodecades. Consider a $(6 \times 2)$-grid as follows:

A dodecade of $\hat{\mathcal{X}}$ is an induced subgraph of $\hat{\Gamma}$ with 12 vertices

$$
D=\left\{a^{1}, b^{1}, c^{1}, d^{1}, e^{1}, f^{1}, a^{2}, b^{2}, c^{2}, d^{2}, e^{2}, f^{2}\right\}
$$

isomorphic to the complement of the collinearity graph of a grid as in figure 3. Clearly, $D$ admits a bipartition into two classes of size 6 (corresponding to the two long lines of the grid) as well as a partition into six 2-sets, corresponding to the six short lines. Two points in the same 2-set are said to be opposite. Clearly, any four (respectively, three) pairs of opposite vertices of a dodecade form a cube (respectively, a hexagon). We say that a dodecade is centric if all the projective lines through opposite vertices are concurrent. The point of concurrence is called the center of the dodecade.

Let $\varphi=\varphi_{0} \circ \hat{\alpha}$, where $\varphi_{0}: \hat{\mathcal{X}} \rightarrow \mathcal{X}$ is the canonical 2-fold covering of $\mathcal{X}$ as in Theorem 1 and $\hat{\alpha} \in \operatorname{Aut}(\hat{\mathcal{X}})$.

For the subgraph $\Gamma_{A}$ of $\Gamma$ induced on a subset $A$ of the vertex set of $\mathcal{X}$, the subgraph $\hat{\Gamma}_{A}$ of $\hat{\Gamma}$ induced on the subset $\varphi^{-1}(A)$ of the vertex set of $\hat{\Gamma}$ is called the preimage of $\Gamma_{A}$ by $\varphi$.

Given a path $\left(X_{0}, X_{1}, \ldots, X_{n}\right)$ in $\Gamma$ and a vertex $x_{0} \in \varphi^{-1}\left(X_{0}\right)$, there exists a unique path $\left(x_{0}, x_{1}, \ldots, x_{n}\right)$ in $\hat{\Gamma}$ such that $\varphi\left(x_{i}\right)=X_{i}$ for every $i=0,1, \ldots, n$. In graph theory and topology, the path $\left(x_{0}, x_{1}, \ldots, x_{n}\right)$ is called the lifting of $\left(X_{0}, X_{1}, \ldots, X_{n}\right)$ at $x_{0}$ through $\varphi$. By a harmless abuse of terminology, we adapt this terminology 


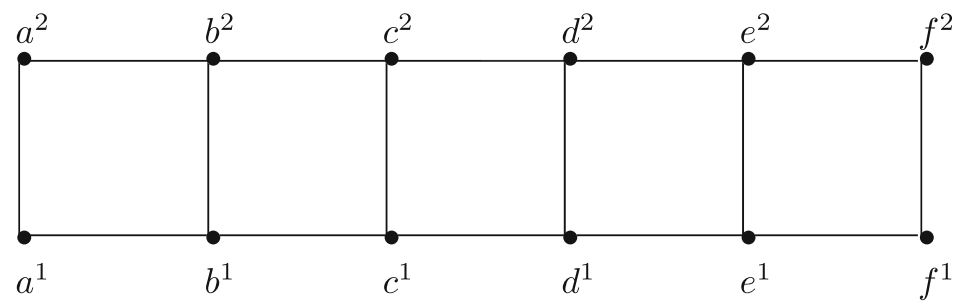

Figure 3. $(6 \times 2)$-grid as complement of a dodecade.

to the induced subgraph $\Gamma_{A}$ of $\Gamma$, calling liftings of $\Gamma_{A}$ the connected components of $\hat{\Gamma}_{A}$.

The following theorem characterizes the cliques of $\Gamma$ in terms of their liftings. Recall that $n_{0}$ is the nucleus of $Q_{0}$.

Theorem 3. Suppose $q \geq 4$. Let $\varphi=\varphi_{0} \circ \hat{\alpha}$ with $\varphi_{0}$ and $\hat{\alpha}$ as above. The following hold:

(a) The covering $\varphi$ induces a bijection between the set of all centric hexagons of $\hat{\Gamma}$ with center $n_{0}$ and the set of all non-linear 3-cliques in $\Gamma$.

(b) The covering $\varphi$ induces a bijection between the set of all centric cubes of $\hat{\Gamma}$ with center $n_{0}$ and the set of all non-linear 4-cliques in $\Gamma$.

(c) The covering $\varphi$ induces a bijection between the set of all centric dodecades of $\hat{\Gamma}$ with center $n_{0}$ and the set of all non-linear 6-cliques in $\Gamma$.

Let $S$ be a subset of $\mathrm{PG}(V)$. According to Theorem 1.1 of [3], there exists a unique subfield $\mathbb{F}_{S}$ of $\mathbb{F}_{q}$ such that $S$ is contained in a projective subgeometry of $\operatorname{PG}(V)$ defined over $\mathbb{F}_{S}$ and is minimal with respect to this property. Moreover, the family of projective subgeometries of $\mathrm{PG}(V)$ defined over $\mathbb{F}_{S}$ and containing $S$ admits a smallest member which we shall denote by $\langle S\rangle_{\mathbb{F}_{S}}$. We call $\langle S\rangle_{\mathbb{F}_{S}}$ the $\mathbb{F}_{S}$-span of $S$.

If $S$ is a subset of $Q$, we define the subgeometry of $Q$ induced on $S$ to be the pair $Q(S):=\left(S, \mathcal{L}_{S}\right)$, where $\mathcal{L}_{S}:=\{l \cap S: l$ is a line of $Q$ such that $|l \cap S| \geq 2\}$.

As recalled a few lines above, there exists a unique smallest projective subgeometry $\langle S\rangle_{\mathbb{F}_{S}}$ of $\mathrm{PG}(V)$ containing $S$ and defined over a subfield $\mathbb{F}_{S}$ of $\mathbb{F}_{q}$. We say that $Q(S)$ is an $\mathbb{F}_{2}$-subgeometry of $Q$ if $\mathbb{F}_{S}=\mathbb{F}_{2}$.

The next theorem, combined with Theorem 3, gives a characterization of non-linear $i$-cliques, $i \in\{3,4,6\}$, in terms of $\mathbb{F}_{2}$-subgeometries of $Q$.

Theorem 4. Suppose $q \geq 4$. The following hold:

(a) A centric hexagon of $\hat{\mathcal{X}}$ with center $n_{0}$ is the subgraph of $\hat{\Gamma}$ induced on the setcomplement $Q(S) \backslash H_{0}$ of $H_{0}=n_{0}^{\perp}$ in an $\mathbb{F}_{2}$-subgeometry $Q(S) \cong Q^{+}(3,2)$ of $Q$ such that $n_{0} \in\langle S\rangle_{\mathbb{F}_{2}}$.

(b) A centric cube of $\hat{\mathcal{X}}$ with center $n_{0}$ is the subgraph of $\hat{\Gamma}$ induced on the setcomplement $Q(S) \backslash H_{0}$ of $H_{0}=n_{0}^{\perp}$ in an $\mathbb{F}_{2}$-subgeometry $Q(S) \cong Q(4,2)$ of $Q$ such that $n_{0} \in\langle S\rangle_{\mathbb{F}_{2}}$.

(c) A centric dodecade of $\hat{\mathcal{X}}$ with center $n_{0}$ is the subgraph of $\hat{\Gamma}$ induced on the setcomplement $Q(S) \backslash H_{0}$ of $H_{0}=n_{0}^{\perp}$ in a $\mathbb{F}_{2}$-subgeometry $Q(S) \cong Q^{-}(5,2)$ of $Q$ such that $n_{0} \in\langle S\rangle_{\mathbb{F}_{2}}$. 
The relevance of the parity of $n$ for the existence of maximal non-linear 6-cliques in Theorem 2 is justified by Theorems 3 and 4 . Indeed, every non-linear 6-clique of $\Gamma$ arises as the complement of $H_{0}$ in a $(2,4)$-subquadrangle $Q^{-}(5,2)$ of $Q$ (Theorem 4(c)), but it is an easy exercise to show that $Q^{-}(5,2)$ is a subgeometry of $Q^{-}\left(5,2^{n}\right)$ if and only if $n$ is odd.

Each non-linear 4-clique of $\Gamma$ arises as the complement of a tangent hyperplane in the $(2,2)$-subquadrangle $Q(4,2)$ of $Q^{-}\left(5,2^{n}\right)$. Observe that $Q(4,2)$ is a subgeometry of $Q^{-}\left(5,2^{n}\right)$ for each $n$. So, non-linear 4-cliques exist for all $n$.

\section{The semi-partial geometry $\mathcal{X}$}

We keep the terminology and notation of $\S 1$.

\subsection{The quotient geometry $\hat{\mathcal{X}} / R$}

Let $R$ be the equivalence relation on the point set $\hat{P}$ of the affine generalized quadrangle $\hat{\mathcal{X}}$ defined as follows: for $x, y \in \hat{P}$, define $x R y$ if and only if either $x=y$ or $x^{\perp} \cap Q_{0}=$ $y^{\perp} \cap Q_{0}\left(=x^{\perp} \cap y^{\perp}\right)$, where $\perp$ is the orthogonality relation defined by $Q$, as in $\S 1.1$ (see $\S 8.4$ of [9]). Note that distinct points of $\mathcal{X}$ are $R$-equivalent if and only if they have distance 3 in the collinearity graph $\hat{\Gamma}$ of $\hat{\mathcal{X}}$.

For $x \in \hat{P}$ and $\hat{l} \in \hat{L}$, let $[x]=\{y \in \hat{P}: x R y\}$ and $[\hat{l}]:=\{[x]: x \in \hat{l}\}$. Note that $|[x]|=2$ and $|[\hat{l}]|=q$. Given any two distinct lines $\hat{l}$ and $\hat{m}$ of $\hat{\mathcal{X}}$, we put $\hat{l} R \hat{m}$ if and only if $[\hat{l}]=[\hat{m}]$.

We define the quotient geometry $\hat{\mathcal{X}} / R$ as the point-line geometry having $\{[x]: x \in \hat{P}\}$ as the point set and $\{[\hat{l}]: \hat{l} \in \hat{L}\}$ as the line set. A point $[x]$ is incident with a line $[\hat{l}]$ if $[x] \in[\hat{l}]$.

We now give an equivalent description of the quotient geometry $\hat{\mathcal{X}} / R$. We recall some well-known facts about the action of the polarity defined by $\perp$ on the subspaces of $\mathrm{PG}(V)$ (see, e.g., Chapter 22.3 of [6]).

- If $x$ is a point of $Q$, then $x^{\perp}$ is a hyperplane tangent to $Q$ at $x$.

- If $S$ is a 3-dimensional subspace of $\operatorname{PG}(V)$ such that $S \cap Q$ is an elliptic quadric in $S$, then $S^{\perp}$ is a line secant to $Q$.

- If $S$ is a 3-dimensional subspace of $\operatorname{PG}(V)$ such that $S \cap Q$ is a hyperbolic quadric in $S$, then $S^{\perp}$ is a line disjoint from $Q$.

- If $\pi_{p}$ is a projective plane of $\operatorname{PG}(V)$ such that $\pi_{p} \cap Q=\{p\}$, then $\pi_{p}^{\perp}$ is a projective plane intersecting $Q$ in two lines meeting at $p$. Moreover, $\pi_{p} \cap \pi_{p}^{\perp}=\{p\}$ and $\left\langle\pi_{p}, \pi_{p}^{\perp}\right\rangle=p^{\perp}$.

Let $v$ be the unique non-trivial elation of $\operatorname{PG}(V)$ stabilizing $Q$ and having the nucleus $n_{0}$ of $Q_{0}$ as the center and the hyperplane $H_{0}=n_{0}^{\perp}$ as the axis. Clearly, $v$ is an involution. The orbits of $v$ on the point set of $\hat{\mathcal{X}}$ are precisely the equivalence classes of $R$ on $\hat{P}$. Hence, for any point $x$ of $\hat{P}$, the $R$-equivalence class $[x]$ is $\{x, v(x)\}$ obtained as $\left\langle x, n_{0}\right\rangle \cap$ $Q$, where $\left\langle x, n_{0}\right\rangle$ is the line (secant to $Q$ ) of $\mathrm{PG}(V)$ through $x$ and $n_{0}$. Accordingly, a line $[\hat{l}]$ of $\hat{\mathcal{X}} / R$ corresponds to the set $(l \cup v(l)) \backslash(l \cap v(l))$ obtained as $\langle l, v(l)\rangle \cap Q$ minus the point $l \cap v(l)$, where $l=\hat{l} \cup\left\{l^{\infty}\right\}$ (see (1)). Note also that $l \cap \nu(l)=l^{\infty}$ and $\langle l, v(l)\rangle=\left\langle l, n_{0}\right\rangle$.

\subsection{Proof of Theorem 1}

According to the definition of $t$-fold covering given in $\$ 1.1$ and the facts recalled in $\S 2.1$, it is easy to see that the projection $\pi: \hat{\mathcal{X}} \rightarrow \hat{\mathcal{X}} / R$ mapping any point $x$ of $\hat{\mathcal{X}}$ to the point $[x]$ of $\hat{\mathcal{X}} / R$ and any line $l$ of $\hat{\mathcal{X}}$ to the line $[l]$ of $\hat{\mathcal{X}} / R$ is a 2 -fold covering. Hence 
Lemma 2.1. $\hat{\mathcal{X}}$ is a 2 -fold cover of $\hat{\mathcal{X}} / R$.

Lemma 2.2. The geometries $\hat{\mathcal{X}} / R$ and $\mathcal{X}$ are isomorphic.

Proof. Let $\psi: \mathcal{X} \rightarrow \hat{\mathcal{X}} / R$ be the map defined on the points and the lines of $\mathcal{X}$ as follows: for $X \in \mathcal{E}, X^{\perp}$ is a line of $\operatorname{PG}(5, q)$ through $n_{0}$, secant to $Q$. We set $\psi(X)=X^{\perp} \cap Q$. For a line $\rho_{p}$ of $\mathcal{X}$, let $\pi_{p}$ be the unique tangent plane to all $X \in \rho_{p}$ (see Proposition 1.2). Then, $\pi_{p}{ }^{\perp}$ is a plane of $\operatorname{PG}(5, q)$ intersecting $Q$ in two lines $l, l^{\prime}$ such that $l \cap Q_{0}=$ $l^{\prime} \cap Q_{0}=l \cap l^{\prime}=\{p\}$. Then, $\psi\left(\rho_{p}\right)=[\hat{l}]=\left[\hat{l}^{\prime}\right]$. It is easily seen that $\psi$ is an isomorphism of geometries.

Theorem 1 follows from Lemmas 2.1 and 2.2, in particular, $\varphi_{0}:=\psi^{-1} \circ \pi$ where $\varphi_{0}$ is the canonical covering of $\mathcal{X}$ as defined in Theorem 1.

\section{The collinearity graph of $\hat{\mathcal{X}}$}

In this section we will investigate properties of the collinearity graph $\Gamma$ of $\mathcal{X}$. Recall that the vertices of $\Gamma$ are the elliptic ovoids of $Q_{0}$ and two vertices $X_{1}$ and $X_{2}$ are adjacent if and only if they are tangent, i.e. $\left|X_{1} \cap X_{2}\right|=1$.

If $q=2$, then $\Gamma$ is a complete graph on 6 vertices, $\hat{\Gamma}$ is the set complement of a $(2 \times 6)$-grid and the $R$-equivalence classes are the short lines of the grid.

From now on, we assume that $q \geq 4$.

\section{PROPOSITION 3.1}

If $q>2$, then the collinearity graph $\Gamma$ of $\mathcal{X}$ has diameter 2.

Proof. The collinearity graph $\hat{\Gamma}$ of $\hat{\mathcal{X}}$ has diameter 3 and the $R$-equivalence classes are the pairs of points of $\hat{\mathcal{X}}$ at mutual distance 3 (see $\S 8.4$ of [9]). Hence $\hat{\Gamma} / R$ has diameter at most 2. In fact, it is easy to see that it has diameter exactly 2 except when $q=2$. By Lemma 2.2, the graph $\Gamma$ has diameter 2.

In [7], it is proved that $\Gamma$ is a strongly regular graph with parameters $v=q^{2}\left(q^{2}-1\right) / 2$, $k=(q-1)\left(q^{2}+1\right), \lambda=(q-1)(q+2)$ and $\mu=2 q(q-1)$.

Relying on properties of the canonical covering $\varphi_{0}$, we can immediately deduce the parameters $(v, k, \lambda, \mu)$ of $\Gamma$ and hence, in light of Proposition 3.1, reprove that $\Gamma$ is a strongly regular graph. Indeed,

$v$ : The number of vertices of $\Gamma$ is $|\hat{P}| / 2$.

$k$ : The number of vertices adjacent to a given vertex $X$ of $\Gamma$ is $\mid\{$ lines of $\hat{\mathcal{X}}$ through $x\} \mid \times$ $\mid$ ppoints different from $x$ on any line of $\hat{\mathcal{X}}$ through $x\} \mid$, for a point $x \in X^{\perp} \cap \hat{P}$.

$\lambda$ : Let $X$ and $Y$ be two adjacent vertices of $\Gamma$, i.e., $X$ and $Y$ are two elliptic ovoids of $Q_{0}$ tangent at some point $p \in Q_{0}$. They define a line $\rho_{p}$ of $\mathcal{X}$. Then, $\varphi_{0}^{-1}\left(\rho_{p}\right)=\pi_{p}^{\perp} \cap Q=$ $\left\{l^{1}, l^{2}\right\} \backslash\{p\}$, where $l^{1}, l^{2}$ are lines of $Q$ through $p, \varphi_{0}^{-1}(X)=\left\{x^{1}, x^{2}\right\} \subset \varphi_{0}^{-1}\left(\rho_{p}\right)$ and $\varphi_{0}^{-1}(Y)=\left\{y^{1}, y^{2}\right\} \subset \varphi_{0}^{-1}\left(\rho_{p}\right)$. Suppose that $x^{1}$ and $y^{1}$ are on $\hat{l}^{1}$ and that $x^{2}$ and $y^{2}$ are on $\hat{l}^{2}$. The number of vertices of $\Gamma$ adjacent to both $X$ and $Y$ is the same as the number $q-2$ of points $z \in \hat{P}$ on $\hat{l}^{1} \backslash\left\{x^{1}, y^{1}\right\}$ (equivalently, the number of points on $\hat{l}^{2} \backslash\left\{x^{2}, y^{2}\right\}$ ). 
These points bijectively correspond to the ovoids of $\rho_{p}$ different from $X$ and $Y$. We also have to count the number of vertices of $\Gamma$ adjacent to both $X$ and $Y$ but not on the same line $\rho_{p}$ of $\mathcal{X}$ defined by $X$ and $Y$. This number is equal to the number of points $z \in \hat{\mathcal{X}}$ at distance 1 from both $x^{1}$ and $y^{2}$ (equivalently, at distance 1 from both $x^{2}$ and $y^{1}$ ). There are $q^{2}$ such points. Indeed, on any line of $\hat{\mathcal{X}}$ through $x^{1}$ different from $\hat{l}^{1}$, there is a unique point of $\hat{\mathcal{X}}$ collinear to $y^{2}$. Hence, $\lambda=q^{2}+q-2$.

$\mu$ : Let $X$ and $Y$ be two non-adjacent vertices of $\Gamma$, i.e., $X$ and $Y$ are two elliptic ovoids of $Q_{0}$ intersecting in a conic. Suppose $\varphi_{0}^{-1}(X)=\left\{x^{1}, x^{2}\right\}$ and $\varphi_{0}^{-1}(Y)=\left\{y^{1}, y^{2}\right\}$. Note that there are no elliptic ovoids $Z$ tangent to both $X$ and $Y$ at a point in $X \cap Y$ because, otherwise, $X$ and $Y$ would be on the same line of $\mathcal{X}$, which is not possible because $X$ and $Y$ are not adjacent in $\Gamma$. Thus, the number of vertices of $\Gamma$ adjacent to both $X$ and $Y$ is the same as the number of points $z \in Q \backslash Q_{0}$ not collinear with any point of $X \cap Y$, but collinear to $x^{1}$ and $y^{2}$ or to $x^{2}$ and $y^{1}$. Let $\hat{l}$ be a line of $\hat{\mathcal{X}}$ through $y^{1}$ with $l^{\infty} \in Y \backslash X$. There are $q^{2}-q$ such lines. For each of these $q^{2}-q$ lines, there are exactly two distinct points adjacent to only one of $x^{1}$ or $x^{2}$. Hence $\mu=2\left(q^{2}-q\right)$.

In the above calculation of the parameters $\lambda$ and $\mu$, we have also proved two interesting properties of the geometry $\mathcal{X}$ which are stated as part (c) and (d) of Proposition 1.1.

\subsection{Cliques of $\Gamma$ and their liftings}

In this section we classify cliques in $\Gamma$. According to $\S 1.1$, a clique in $\Gamma$ consisting of members of $\mathcal{X}$ sharing the same point $p$ is a linear clique (based at $p$ ). A rosette of $\mathcal{X}$ based at $p$, being a maximal pencil of elliptic ovoids mutually tangent at $p$, is a maximal linear clique (this is part of Claims (a) and (b) of Theorem 2).

Let $\hat{\Gamma}$ denote the collinearity graph of the affine quadrangle $\hat{\mathcal{X}}$, as in $\S 1$. Let $\varphi_{0}: \hat{\mathcal{X}} \rightarrow$ $\mathcal{X}$ be the canonical covering as in Theorem 1. The fibers of $\varphi_{0}$ are the pairs of points of $\hat{\mathcal{X}}$ at mutual distance 3 (see $\S 2.1$ ). We will adopt the following notation. If $X$ is a vertex of $\Gamma$, then $\varphi^{-1}(X)=\left\{x^{1}, x^{2}\right\}\left(=X^{\perp} \cap Q\right)$.

\subsection{Preimages of edges and linear cliques}

The preimage of an edge in $\Gamma$ is the union of two disjoint edges in $\hat{\Gamma}$ which are the liftings of that edge. Given a line $\rho_{p}$ of $\mathcal{X}$, we have $\varphi_{0}^{-1}\left(\rho_{p}\right)=\hat{l} \cup \hat{m}$ for two lines $\hat{l}, \hat{m}$ of $\hat{\mathcal{X}}$ such that $l^{\infty}=m^{\infty}=p$. The preimage of the subgraph $\Gamma_{\rho_{p}}$ induced by $\Gamma$ on $\rho_{p}$ is the subgraph induced by $\hat{\Gamma}$ on $\hat{l} \cup \hat{m}$. This graph is the disjoint union of two complete subgraphs, namely $\hat{l}$ and $\hat{m}$.

\section{PROPOSITION 3.2}

Let $\mathcal{C}$ be an $i$-clique of $\Gamma, i \geq 4$, containing a linear 3-clique. Then $\mathcal{C}$ is a linear clique.

Proof. Let $A, B, C, X$ be four distinct vertices of $\mathcal{C}$, with $A, B, C$ forming a linear subclique and let $\rho$ be the line of $\mathcal{X}$ on $\{A, B, C\}$.

By way of contradiction, suppose that $X \notin \rho$. Let us lift the ordered closed configuration $(X, A, B, C, X)$ of $\Gamma$ starting at $x^{1}$ recalling that $A, B, C$ are on the same line $\rho$ of $\mathcal{X}$. Since $\varphi_{0}$ is a covering, the point $x^{1}$ is adjacent in $\hat{\Gamma}$ with exactly one preimage of $A$, say $a^{1}$. Similarly, $a^{1}$ is adjacent with exactly one preimage $b^{1}$ of $B$ and $b^{1}$ is adjacent 
with exactly one preimage $c^{1}$ of $C$. Again, $c^{1}$ is adjacent with exactly one preimage $x^{2}$ of $X$. Moreover, since $\varphi_{0}$ is a covering, $\varphi_{0}^{-1}(\rho)$ contains exactly one line $\hat{l}$ of $\hat{\mathcal{X}}$ incident with all of $a^{1}, b^{1}, c^{1}$. On the other hand, $x^{1}, x^{2} \notin \hat{l}$ as $X \notin \rho$.

Since there are no triangles in $\hat{\mathcal{X}}, x^{1} \neq x^{2}$. The path $\left(x^{1}, a^{1}, b^{1}, c^{1}, x^{2}\right)$ is the lifting of $(X, A, B, C, X)$ at $x^{1}$.

Lift now the closed ordered path $(X, A, B, X)$ of $\Gamma$ starting at $x^{1}$. Proceeding in the same way as above, we get a path $\left(x^{1}, a^{1}, b^{1}, x^{3}\right)$ where the vertex $x^{3}$ must be different from $x^{1}$ because there are no triangles in $\hat{\mathcal{X}}$. Similarly, $\left(x^{2}, c^{1}, b^{1}, x^{3}\right)$ is the lifting of $(X, C, B, X)$ at $x^{2}$. Hence $x^{3} \neq x^{2}$ (see figure 4 ).

It follows that $\varphi_{0}^{-1}(X)$ contains at least three distinct points, namely $x^{1}, x^{2}, x^{3}$. This is a contradiction because $\varphi_{0}$ is a 2 -fold covering.

\subsection{Liftings of non-linear cliques}

We establish the following notation for projective points: $a=[v]=\left[v_{1}, \ldots, v_{n}\right]$, where $v=\left(v_{1}, \ldots, v_{n}\right)$ is a given vector representing the projective point $a$.

Given two projective points $a$ and $b$, symbols such as $t a+s b$ with $t$ and $s$ scalars are nonsense. However, when particular representatives $v$ and $w$ have been chosen for $a$ and $b$, we will take the liberty of writing $t a+s b$ for $[t v+s w]$. Similarly, given a quadratic form $f$, we write $f(a)$ for $f(v)$. Of course, these are notational abuses but they are harmless as far as it is clear which is the representative vector chosen for a given point. In particular, when doing so, we forbid ourselves any rescaling of the given vectors.

Suppose that the $\left(q, q^{2}\right)$-generalized quadrangle $Q$ is represented, with respect to a given basis, by the equation $f(v)=0$, where the coordinates of $v$ with respect to the given basis are $\left(x_{i}\right)_{i=1}^{6}$ and

$$
f\left(\left(x_{1}, x_{2}, x_{3}, x_{4}, x_{5}, x_{6}\right)\right):=x_{1} x_{2}+x_{3} x_{4}+x_{5}^{2}+x_{5} x_{6}+\lambda x_{6}^{2}
$$

with $\lambda$ a given element of $\mathbb{F}_{q}$ with $\operatorname{Tr}(\lambda)=1$. Recall that the trace function $\operatorname{Tr}: \mathbb{F}_{q} \rightarrow \mathbb{F}_{2}$, $\operatorname{Tr}(x)=\sum_{i=0}^{n-1} x^{2^{i}},\left(q=2^{n}\right)$ is an $\mathbb{F}_{2}$-linear surjective homomorphism and a polynomial $x^{2}+x y+\lambda y^{2}$ is irreducible over $\mathbb{F}_{q}$ if and only if $\operatorname{Tr}(\lambda)=1$.

The nucleus $n_{0}$ of $Q_{0}$ is represented by the vector $(0,0,0,0,0,1)$. Accordingly, $H=$ $n_{0}^{\perp}$ is the hyperplane defined by the equation $x_{6}=0$.

We first prove a technical lemma.

Lemma 3.3. Let $\lambda, \mu$ be two elements of $\mathbb{F}_{q}$. Suppose that $\operatorname{Tr}(\lambda)=1$ and $\mu \neq 1$. Then the equation $x^{2}+x y+\lambda y^{2}+\mu=1$, in the unknowns $x, y$, admits $q+1$ distinct solutions in $\mathbb{F}_{q}^{2}$.

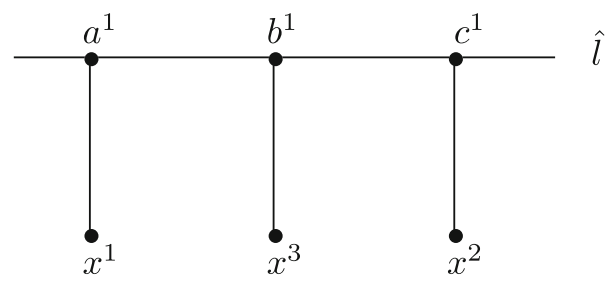

Figure 4. Argument in the proof of Proposition 3.2. 
Proof. If $y=0$, then the pair $(x, y)=\left(\mu^{2^{n-1}}+1,0\right)$ is the unique solution of $x^{2}+x y+$ $\lambda y^{2}+\mu=1$. If $y \neq 0$, then the equation $x^{2}+x y+\lambda y^{2}+\mu=1$ is soluble if and only if the equation $t^{2}+t+\lambda+(\mu+1) / y^{2}=0$ is soluble in the unknown $t:=x / y$. This latter requirement is equivalent to $\operatorname{Tr}\left(\lambda+(\mu+1) / y^{2}\right)=0$. Since

$$
\operatorname{Tr}\left(\lambda+(\mu+1) / y^{2}\right)=\operatorname{Tr}(\lambda)+\operatorname{Tr}\left((\mu+1) / y^{2}\right)=1+\operatorname{Tr}\left((\mu+1) / y^{2}\right)
$$

the equation $t^{2}+t+\left(\lambda+(\mu+1) / y^{2}\right)=0$ admits solutions if and only if $\operatorname{Tr}\left((\mu+1) / y^{2}\right)=1$.

As the trace function $\mathrm{Tr}$ is a surjective homomorphism, there are $q / 2$ distinct values of $y$ such that $\operatorname{Tr}\left((\mu+1) / y^{2}\right)=1$ (see e.g. [8]). For each of these $q / 2$ values of $y$, there are two distinct solutions of $t^{2}+t+\lambda+(\mu+1) / y^{2}=0$. So, if $y \neq 0$, there are $2 \cdot \frac{q}{2}$ distinct pairs $(x, y)$ which satisfy $x^{2}+x y+\lambda y^{2}+\mu=1$.

3.3.1 Non-linear $i$-cliques, $i \in\{3,4\}$. Hexagons, cubes and dodecades of $\hat{\mathcal{X}}$ and their centers, if any, have been defined in $§ 1.1$. Clearly, those definitions can be immediately generalized to $Q$, by replacing $\hat{\Gamma}$ with the collinearity graph of $Q$. In particular, a hexagon (cube, dodecade) of $Q$ is centric if the projective lines joining pairs of opposite points are concurrent in one point, called the center of the hexagon (cube, dodecade). These more general notions will be used from time to time in the sequel.

Theorem 3.4. The preimage in $\hat{\Gamma}$ of a non-linear 3-clique of $\Gamma$ under $\varphi_{0}$ is a centric hexagon of $\hat{\mathcal{X}}$ with the nucleus $n_{0}$ of $Q_{0}$ as the center.

Proof. Let $\mathcal{C}=\{A, B, C\}$ be a non-linear 3-clique of $\Gamma$. The lifting of the cycle $(A, B, C, A)$ of $\mathcal{C}$ starting at $a^{1} \in \varphi_{0}^{-1}(A)$ is the path $\left(a^{1}, b^{1}, c^{1}, a^{2}\right)$ of $\hat{\Gamma}$. Since $\hat{\mathcal{X}}$ has no proper triangles, $a^{1} \neq a^{2}$. The lifting of $(A, B, C, A)$ at $a^{2}$ is the path $\left(a^{2}, b^{2}, c^{2}, a^{3}\right)$, where $b^{2} \neq b^{1}$ and $c^{2} \neq c^{1}$ because there are no proper triangles in $\hat{\mathcal{X}}$ and $a^{3}$ is a preimage of $A$, different from $a^{2}$. As $\left|\varphi_{0}^{-1}(A)\right|=2$, necessarily $a^{3}=a^{1}$. Now, by pasting $\left\{a^{1}, b^{1}, c^{1}, a^{2}\right\}$ with $\left\{a^{2}, b^{2}, c^{2}, a^{1}\right\}$ we obtain a proper hexagon $H$. As $\varphi_{0}$ is a 2 -fold covering, $H=\varphi_{0}^{-1}(\mathcal{C})$, that is, $\mathcal{C}$ lifts to $H$.

By definition of $\varphi_{0}$ (see Theorem 1), each of the pairs $\left\{a^{1}, a^{2}\right\},\left\{b^{1}, b^{2}\right\},\left\{c^{1}, c^{2}\right\}$ is on a line secant to $Q$ through $n_{0}$.

Every centric hexagon of $\hat{\Gamma}$ with center $n_{0}$ is mapped by $\varphi_{0}$ onto a non-linear 3 -clique of $\Gamma$. This, combined with Theorem 3.4, proves Theorem 3(a).

Theorem 3.5. The preimage of a non-linear 4-clique of $\Gamma$ under $\varphi_{0}$ is a centric cube of $\hat{\mathcal{X}}$ with the nucleus $n_{0}$ of $Q_{0}$ as the center.

Proof. Let $\mathcal{C}=\{A, B, C, D\}$ be a non-linear 4-clique of $\Gamma$. Let $\varphi_{0}^{-1}(A)=\left\{a^{1}, a^{2}\right\}$, $\varphi_{0}^{-1}(B)=\left\{b^{1}, b^{2}\right\}, \varphi_{0}^{-1}(C)=\left\{c^{1}, c^{2}\right\}$ and $\varphi_{0}^{-1}(D)=\left\{d^{1}, d^{2}\right\}$.

The lifting of the closed path $(A, B, C, D, A)$ of $\mathcal{C}$ starting at $a^{1}$ is a path $\mathcal{C}^{1}=$ $\left(a^{1}, b^{1}, c^{1}, d^{1}, a^{i}\right)$, where $i=1$ or $i=2$. Suppose $i=2$. Then, the lifting of the closed path $(A, B, C, A)$ starting at $a^{1}$ is either $\left(a^{1}, b^{1}, c^{1}, a^{1}\right)$ or $\left(a^{1}, b^{1}, c^{1}, a^{2}\right)$. None 
of the latter liftings is possible, else we get a proper triangle in $\hat{\mathcal{X}}$. Hence $i=1$, namely $\mathcal{C}^{1}=\left(a^{1}, b^{1}, c^{1}, d^{1}, a^{1}\right)$.

Since $\hat{\mathcal{X}}$ has no proper triangles, the following hold:

(a) the lifting of $(A, B, C, A)$ starting at $a^{1}$ is $\left(a^{1}, b^{1}, c^{1}, a^{2}\right)$;

(b) the lifting of $(A, C, D, A)$ starting at $a^{1}$ is $\left(a^{1}, c^{2}, d^{2}, a^{2}\right)$;

(c) the lifting of $(B, C, A, B)$ starting at $b^{1}$ is $\left(b^{1}, c^{1}, a^{2}, b^{2}\right)$;

(d) the lifting of $(B, D, A, B)$ starting at $b^{1}$ is $\left(b^{1}, d^{2}, a^{2}, b^{2}\right)$;

(e) the lifting of $(D, B, C, D)$ starting at $d^{1}$ is $\left(d^{1}, b^{2}, c^{2}, d^{2}\right)$.

Thus, we get the cube as in figure 2 .

By definition of $\varphi_{0}$, each of the pairs $\left\{a^{1}, a^{2}\right\},\left\{b^{1}, b^{2}\right\},\left\{c^{1}, c^{2}\right\},\left\{d^{1}, d^{2}\right\}$ is on a line secant to $Q$ through $n_{0}$.

Every centric cube of $\hat{\Gamma}$ with center $n_{0}$ is mapped by $\varphi_{0}$ onto a non-linear 4-clique of $\Gamma$. This, combined with Theorem 3.5, proves Theorem 3(b).

A set of four distinct points $\{a, b, c, d\}$ of $Q$ is a quadrangle of $Q$ if it is the set of points of a $(1,1)$-subquadrangle of $Q$, that is,

$$
f(a)=f(b)=f(c)=f(d)=0 ; a \perp b \perp c \perp d \perp a ; a \not \perp c ; \quad b \not \perp d .
$$

Let $\mathcal{F}$ be a quadrangle of $Q$. We denote by $I(\mathcal{F})$ the set of points $p \in \operatorname{PG}(V) \backslash Q$ such that $p^{\perp} \cap \mathcal{F}=\emptyset$ and there exists a cube in the affine generalized quadrangle $Q \backslash p^{\perp}$ with center $p$ having $\mathcal{F}$ as one of its faces. Clearly, such a cube is uniquely determined by $\mathcal{F}$ and $p$.

Lemma 3.6. Let $\overline{\mathcal{F}}=\left\{\bar{a}^{1}, \bar{b}^{1}, \bar{c}^{1}, \bar{d}^{1}\right\}$ be the quadrangle of $Q$ with

$$
\begin{aligned}
& \bar{a}^{1}=[1,0,0,0,0,0], \bar{b}^{1}=[0,0,1,0,0,0], \\
& \bar{c}^{1}=[0,1,0,0,0,0], \bar{d}^{1}=[0,0,0,1,0,0] .
\end{aligned}
$$

Then,

$$
I(\overline{\mathcal{F}})=\left\{\left[u, \frac{1}{u}, v, \frac{1}{v}, r, s\right]: r^{2}+r s+\lambda s^{2}=1, u v \neq 0, u, v \in \mathbb{F}_{q}\right\}
$$

and

$$
|I(\overline{\mathcal{F}})|=(q-1)^{2}(q+1)=(q-1)\left(q^{2}-1\right) .
$$

Proof. Let $\bar{p}=\left[p_{1}, p_{2}, p_{3}, p_{4}, r, s\right] \in I(\overline{\mathcal{F}})$. Since $\bar{p} \notin Q, f(\bar{p}) \neq 0$ (with $f$ as in (2)). Without loss of generality, we can assume that $f(\bar{p})=1$, i.e.

$$
p_{1} p_{2}+p_{3} p_{4}+r^{2}+r s+\lambda s^{2}=1 .
$$

Since $\bar{p}^{\perp} \cap \overline{\mathcal{F}}=\emptyset$ by definition of $I(\overline{\mathcal{F}}), \bar{p}$ is not collinear in $Q$ with any element of $\overline{\mathcal{F}}$. Hence, in terms of coordinates of $\bar{p}$, we have $p_{1} p_{2} p_{3} p_{4} \neq 0$. Suppose that $\bar{a}^{2}, \bar{b}^{2}, \bar{c}^{2}$, $\bar{d}^{2}$ are points of $Q$ so that $\bar{C}=\left\{\bar{a}^{i}, \bar{b}^{i}, \bar{c}^{i}, \bar{d}^{i}\right\}_{i=1,2}$ is a centric cube of $Q \backslash \bar{p}^{\perp}$ with center $\bar{p}$. Recall that the exponents 1 and 2 of the same symbol refer to opposite points, that the collinearities between points in $\bar{C}$ are as described in figure 2 and each of $\bar{a}^{2}, \bar{b}^{2}, \bar{c}^{2}$, 
$\bar{d}^{2}$ are on a line joining $\bar{p}$ to $\bar{a}^{1}, \bar{b}^{1}, \bar{c}^{1}, \bar{d}^{1}$, respectively. Hence, in terms of coordinates, we have

$$
\bar{a}^{2}=\bar{a}^{1}+t_{a} \bar{p}, \quad \bar{b}^{2}=\bar{b}^{1}+t_{b} \bar{p}, \quad \bar{c}^{2}=\bar{c}^{1}+t_{c} \bar{p}, \quad \bar{d}^{2}=\bar{d}^{1}+t_{d} \bar{p}
$$

for suitable $t_{a}, t_{b}, t_{c}, t_{d} \in \mathbb{F}_{q} \backslash\{0\}$. By hypothesis, plugging in the coordinates of $\bar{a}^{1}, \bar{b}^{1}$, $\bar{c}^{1}, \bar{d}^{1}$, we have

$$
\begin{aligned}
& \bar{a}^{2}=\left[1+t_{a} p_{1}, t_{a} p_{2}, t_{a} p_{3}, t_{a} p_{4}, t_{a} r, t_{a} s\right] ; \\
& \bar{b}^{2}=\left[t_{b} p_{1}, t_{b} p_{2}, 1+t_{b} p_{3}, t_{b} p_{4}, t_{b} r, t_{b} s\right] ; \\
& \bar{c}^{2}=\left[t_{c} p_{1}, t_{c} p_{2}+1, t_{c} p_{3}, t_{c} p_{4}, t_{c} r, t_{c} s\right] ; \\
& \bar{d}^{2}=\left[t_{d} p_{1}, t_{d} p_{2}, t_{d} p_{3}, 1+t_{d} p_{4}, t_{d} r, t_{d} s\right] .
\end{aligned}
$$

Translating in terms of coordinates the collinearities in $Q$ for $\bar{C}$ to be a cube with center $\bar{p}$, we have

$$
\begin{array}{ll}
\bar{a}^{1} \perp \bar{c}^{2}: t_{c} p_{2}+1=0 ; & \bar{b}^{1} \perp \bar{d}^{2}: t_{d} p_{4}+1=0 ; \\
\bar{c}^{1} \perp \bar{a}^{2}: t_{a} p_{1}+1=0 ; & \bar{d}^{1} \perp \bar{b}^{2}: t_{b} p_{3}+1=0 ; \\
\bar{a}^{2} \perp \bar{b}^{2}: t_{b} p_{2}+t_{a} p_{4}=0 ; & \bar{b}^{2} \perp \bar{c}^{2}: t_{b} p_{1}+t_{c} p_{4}=0 ; \\
\bar{c}^{2} \perp \bar{d}^{2}: t_{d} p_{1}+t_{c} p_{3}=0 ; & \bar{d}^{2} \perp \bar{a}^{2}: t_{d} p_{2}+t_{a} p_{3}=0 .
\end{array}
$$

Consequently, $p_{1} p_{2}+p_{3} p_{4}=0$ and (6) becomes

$$
r^{2}+r s+\lambda s^{2}=1 \text {. }
$$

Since $\bar{a}^{2}, \bar{b}^{2}, \bar{c}^{2}, \bar{d}^{2}$ are points of $Q$, we have $f\left(\bar{a}^{2}\right)=f\left(\bar{b}^{2}\right)=f\left(\bar{c}^{2}\right)=f\left(\bar{d}^{2}\right)=0$, i.e., $p_{2}=t_{a}, p_{4}=t_{b}, p_{1}=t_{c}$ and $p_{3}=t_{d}$. The first two rows of (7) give $p_{1} p_{2}=1=p_{3} p_{4}$.

Put $u:=p_{1}$ and $v:=p_{3}$. Then, $\bar{p}=[u, 1 / u, v, 1 / v, r, s]$ where $u v \neq 0$ and $r, s$ satisfy eq. (8).

By Lemma 3.3 with $\mu=0$, (8) admits $q+1$ distinct solutions. So, the lemma follows.

We will refer to the quadrangle $\overline{\mathcal{F}}$ as in Lemma 3.6 as the fundamental quadrangle of $Q$. By the proof of Lemma 3.6, the coordinates of the points of the cube $C_{\bar{p}}$ having $\overline{\mathcal{F}}$ as a face and $\bar{p} \in I(\overline{\mathcal{F}})$ as the center are the following:

$$
\begin{array}{lll}
\bar{a}^{1}=[1,0,0,0,0,0], & \bar{b}^{1}=[0,0,1,0,0,0], \\
\bar{c}^{1}=[0,1,0,0,0,0], & \bar{d}^{1}=[0,0,0,1,0,0], \\
\bar{a}^{2}=\left[0, \frac{1}{u^{2}}, \frac{v}{u}, \frac{1}{u v}, \frac{r}{u}, \frac{s}{u}\right] & \bar{b}^{2}=\left[\frac{u}{v}, \frac{1}{u v}, 0, \frac{1}{v^{2}}, \frac{r}{v}, \frac{s}{v}\right] ; \\
\bar{c}^{2}=\left[u^{2}, 0, u v, \frac{u}{v}, u r, u s\right] & \bar{d}^{2}=\left[u v, \frac{v}{u}, v^{2}, 0, v r, v s\right] \\
\bar{p}=\left[u, \frac{1}{u}, v, \frac{1}{v}, r, s\right], &
\end{array}
$$

where $u, v \in \mathbb{F}_{q}$ with $u v \neq 0$ and $r, s$ satisfies (8). 


\section{COROLLARY 3.7}

Every quadrangle of $Q$ can be extended to $(q-1)^{2}(q+1)$ centric cubes of $Q$.

Proof. This is straightforward, by eq. (5) of Lemma 3.6 and the transitivity of $\mathrm{SO}^{-}(6, q)$ on the set of quadrangles of $Q$.

\section{COROLLARY 3.8}

$\Gamma$ admits non-linear 4-cliques.

Proof. By Theorem 3.5, if a non-linear 4-clique exists, then it can be uniquely lifted to a centric cube with center $n_{0}$. Hence existence of non-linear 4-cliques is equivalent to the existence of centric cubes with center $n_{0}$. By Lemma 3.6, centric cubes having $\overline{\mathcal{F}}$ as a face exist. Let $C_{\bar{p}}$ be one of these cubes and let $\bar{p}$ be its center.

The group $S O^{-}(6, q) \leq \operatorname{Aut}(Q)$ is transitive on the set of points of $P G(5, q) \backslash Q$. Then, there exists $g \in S O^{-}(6, q)$ such that $g(\bar{p})=n_{0}$. The cube $g\left(C_{\bar{p}}\right)$ has the required properties.

We now prove Theorem 2(c).

Theorem 3.9. Every non-linear 3-clique of $\Gamma$ can be extended to $q+1$ non-linear 4cliques of $\Gamma$.

Proof. By Theorems 3.4 and 3.5, each non-linear 3-clique can be extended to $q+1$ nonlinear 4-cliques if and only if each centric hexagon of $\hat{\mathcal{X}}$ can be extended to $q+1$ centric cubes of $\hat{\mathcal{X}}$ having the same center as the hexagon.

Let $H=\left\{a^{i}, b^{i}, c^{i}\right\}_{i=1,2}$ be a centric hexagon of $Q$, with vertices marked as in figure 1 .

Since the group $\mathrm{SO}^{-}(6, q)$ is transitive on the set of paths of length 2 of the collinearity graph of $Q$, we can suppose that $a^{1}, b^{1}, c^{1}$ are indeed the vertices $\bar{a}^{1}, \bar{b}^{1}, \bar{c}^{1}$ as in the fundamental quadrangle $\overline{\mathcal{F}}$, i.e. $a^{1}=[1,0,0,0,0,0], b^{1}=[0,0,1,0,0,0]$ and $c^{1}=$ $[0,1,0,0,0,0]$.

Let us determine the opposite points $a^{2}, b^{2}, c^{2}$. In order to do that, let $p=$ $\left[p_{1}, p_{2}, p_{3}, p_{4}, p_{5}, p_{6}\right]$ be the center of $H$. Hence, $p$ is not collinear in $Q$ with any of $a^{1}, b^{1}, c^{1}$. In terms of coordinates, this fact is equivalent to $p_{1}, p_{2}, p_{3} \neq 0$. Since $p$ is a point of $\mathrm{PG}(V)$ not in $Q, f\left(\left(p_{1}, p_{2}, p_{3}, p_{4}, p_{5}, p_{6}\right)\right) \neq 0$. Without loss of generalities, we can suppose that $f\left(\left(p_{1}, p_{2}, p_{3}, p_{4}, p_{5}, p_{6}\right)\right)=1$, i.e. $p_{1} p_{2}+p_{3} p_{4}+p_{5}^{2}+p_{5} p_{6}+$ $\lambda p_{6}^{2}=1$. Then there exists $\mu_{a}, \mu_{b}, \mu_{c} \in \mathbb{F}_{q} \backslash\{0\}$ such that

$$
\begin{aligned}
a^{2} & =\left[\mu_{a}(1,0,0,0,0,0)+\left(p_{1}, p_{2}, p_{3}, p_{4}, p_{5}, p_{6}\right)\right] \\
& =\left[\mu_{a}+p_{1}, p_{2}, p_{3}, p_{4}, p_{5}, p_{6}\right] \\
b^{2} & =\left[\mu_{b}(0,0,1,0,0,0)+\left(p_{1}, p_{2}, p_{3}, p_{4}, p_{5}, p_{6}\right)\right] \\
& =\left[p_{1}, p_{2}, \mu_{b}+p_{3}, p_{4}, p_{5}, p_{6}\right] \\
c^{2} & =\left[\mu_{c}(0,1,0,0,0,0)+\left(p_{1}, p_{2}, p_{3}, p_{4}, p_{5}, p_{6}\right)\right] \\
& =\left[p_{1}, \mu_{c}+p_{2}, p_{3}, p_{4}, p_{5}, p_{6}\right] .
\end{aligned}
$$

Since $a^{2}, b^{2}, c^{2}$ are points of $Q$ and $f\left(p_{1}, p_{2}, p_{3}, p_{4}, p_{5}, p_{6}\right)=1$ by assumption, we get $\mu_{a}=1 / p_{2}, \mu_{b}=1 / p_{4}, \mu_{c}=1 / p_{1}$, so

$$
\begin{aligned}
& a^{2}=\left[1 / p_{2}+p_{1}, p_{2}, p_{3}, p_{4}, p_{5}, p_{6}\right], \\
& b^{2}=\left[p_{1}, p_{2}, 1 / p_{4}+p_{3}, p_{4}, p_{5}, p_{6}\right], \\
& c^{2}=\left[p_{1}, 1 / p_{1}+p_{2}, p_{3}, p_{4}, p_{5}, p_{6}\right] .
\end{aligned}
$$


Translating in terms of coordinates the collinearity relations in $H$ (see figure 1), we have

$$
\frac{1}{p_{2}}+p_{1}=0 \text { because } a^{2} \perp c^{1} \text { and } \frac{1}{p_{1}}+p_{2}=0 \text { because } c^{2} \perp a^{1} \text {. }
$$

Hence $p_{2}=1 / p_{1}$. Note that the relations $b^{2} \perp a^{2}$ and $b^{1} \perp c^{1}$ are automatically satisfied. Equations (10) become

$$
\begin{aligned}
a^{2} & =\left[0,1 / p_{1}, p_{3}, p_{4}, p_{5}, p_{6}\right], \\
b^{2} & =\left[p_{1}, 1 / p_{1}, 1 / p_{4}+p_{3}, p_{4}, p_{5}, p_{6}\right], \\
c^{2} & =\left[p_{1}, 0, p_{3}, p_{4}, p_{5}, p_{6}\right] .
\end{aligned}
$$

and $p=\left[p_{1}, 1 / p_{1}, p_{3}, p_{4}, p_{5}, p_{6}\right]$ with $p_{3} p_{4}+p_{5}^{2}+p_{5} p_{6}+\lambda p_{6}^{2}=0$.

Let $d^{1}=\left[d_{1}, d_{2}, d_{3}, d_{4}, d_{5}, d_{6}\right]$ and $d^{2}=\mu d^{1}+p\left(\mu \in \mathbb{F}_{q} \backslash\{0\}\right)$ be two points of $Q$. If we require the set $H \cup\left\{d^{1}, d^{2}\right\}$ to be a cube with center $p$, we obtain the following conditions on the coordinates of $d^{1}$ and $d^{2}: d_{2}=0$ because $d^{1} \perp a^{1}$ and $d_{1}=0$ because $d^{1} \perp c^{1}$. Also $d_{4} \neq 0$ since $d^{1} \not \perp b^{1}$. Hence we can assume $d_{4}=1$. Therefore $d^{1}=\left[0,0, d_{3}, 1, d_{5}, d_{6}\right]$. Since $d^{1} \perp b^{2}$, we have

$$
d_{3} p_{4}=\frac{1}{p_{4}}+p_{3}+d_{5} p_{6}+d_{6} p_{5}
$$

Observe that $d^{1} \not \perp b^{1}$. Indeed

$$
\alpha\left(\left(p_{1}, 1 / p_{1}, p_{3}, p_{4}, p_{5}, p_{6}\right),\left(0,0, d_{3}, 1, d_{5}, d_{6}\right)\right)=1 / p_{4} \neq 0
$$

where $\alpha$ is the bilinear form associated to $f$. Since $d^{1}$ is a point of $Q$, we get

$$
d_{3}=d_{5}^{2}+d_{5} d_{6}+\lambda d_{6}^{2}
$$

Let us turn to the opposite point $d^{2}=\mu d^{1}+p$ of $d^{1}$. The fact that $d^{2}$ is a point of $Q$ is equivalent to $1+\mu / p_{4}=0$, hence $\mu=p_{4}$. So,

$$
d^{2}=\left[p_{1}, 1 / p_{1}, p_{4} d_{3}+p_{3}, 0, p_{4} d_{5}+p_{5}, p_{4} d_{6}+p_{6}\right] .
$$

By (12), we get

$$
d^{2}=\left[p_{1}, 1 / p_{1}, p_{5} d_{6}+d_{5} p_{6}+1 / p_{4}, 0, p_{4} d_{5}+p_{5}, p_{4} d_{6}+p_{6}\right] .
$$

Note that the relations $d^{2} \perp b^{1}, d^{2} \perp a^{2}, d^{2} \perp c^{2}$ are automatically satisfied. By (12) and (13) we now have

$$
\left(\frac{1}{p_{4}}\right)^{2}+\frac{p_{3}}{p_{4}}+d_{5} \frac{p_{6}}{p_{4}}+d_{6} \frac{p_{5}}{p_{4}}+d_{5}^{2}+d_{5} d_{6}+\lambda d_{6}^{2}=0
$$

which is equivalent to

$$
\begin{gathered}
\left(d_{5}+\frac{p_{5}}{p_{4}}\right)^{2}+\left(d_{5}+\frac{p_{5}}{p_{4}}\right)\left(d_{6}+\frac{p_{6}}{p_{4}}\right)+\lambda\left(d_{6}+\frac{p_{6}}{p_{4}}\right)^{2} \\
+\left(\frac{p_{5}}{p_{4}}\right)^{2}+\frac{p_{5} p_{6}}{p_{4}^{2}}+\lambda\left(\frac{p_{6}}{p_{4}}\right)^{2}+\left(\frac{1}{p_{4}}\right)^{2}+\frac{p_{3}}{p_{4}}=0 .
\end{gathered}
$$


Since

$$
\begin{gathered}
\left(\frac{p_{5}}{p_{4}}\right)^{2}+\frac{p_{5} p_{6}}{p_{4}^{2}}+\lambda\left(\frac{p_{6}}{p_{4}}\right)^{2}+\left(\frac{1}{p_{4}}\right)^{2}+\frac{p_{3}}{p_{4}} \\
=\frac{1+p_{3} p_{4}+p_{5}^{2}+p_{5} p_{6}+\lambda p_{6}^{2}}{p_{4}^{2}} \\
=\frac{f\left(\left(p_{1}, p_{2}, p_{3}, p_{4}, p_{5}, p_{6}\right)\right)}{p_{4}^{2}}=\frac{1}{p_{4}^{2}} \neq 0,
\end{gathered}
$$

we can apply Lemma 3.3 with $x:=d_{5}+\frac{p_{5}}{p_{4}}, y:=d_{6}+\frac{p_{6}}{p_{4}}, \mu=\frac{1}{p_{4}^{2}}+1$ to obtain $q+1$ different choices for $d^{1}$. The theorem is proved.

3.3.2 Non-linear $i$-cliques, $i \in\{5,6\}$. A decade of $\hat{\mathcal{X}}$ is a subgraph of $\hat{\Gamma}$ with 10 vertices

$$
T=\left\{a^{1}, b^{1}, c^{1}, d^{1}, e^{1}, a^{2}, b^{2}, c^{2}, d^{2}, e^{2}\right\}
$$

isomorphic to the complement of the collinearity graph of a $(5 \times 2)$-grid as shown in figure 5 .

The graph $T$ admits a partition of its vertices by two 5-sets (corresponding to the two long lines of the grid) as well as a partition by five 2-sets (corresponding to the five short lines of the grid). Two points in the same 2-set are said to be opposite. Clearly, any four (respectively, three) 2-sets of opposite vertices of a decade form a cube (respectively, a hexagon). We say that a decade is centric if all the projective lines through opposite vertices are concurrent. The point of concurrence is called the center of the decade.

\section{PROPOSITION 3.10}

\section{The following hold:}

(a) Every non-linear 5-clique of $\Gamma$ lifts through $\varphi_{0}$ to a centric decade in $\hat{\Gamma}$ with the nucleus $n_{0}$ of $Q_{0}$ as the center.

(b) Every non-linear 6-clique of $\Gamma$ lifts through $\varphi_{0}$ to a centric dodecade of $\hat{\Gamma}$ with the nucleus $n_{0}$ of $Q_{0}$ as the center.

Proof. We will only prove (b). The proof of (a) is similar.

Let $\mathcal{C}=\{a, b, c, d, e, f\}$ be a non-linear 6-clique of $\Gamma$. Consider the 4-cliques $\{a, b, c, d\}$ and $\{a, f, e, c\}$ of $\mathcal{C}$ and denote their liftings by $\left\{a^{i}, b^{i}, c^{i}, d^{i}\right\}_{i=1,2}$ and $\left\{a^{i}, f^{i}, e^{i}, c^{i}\right\}_{i=1,2}$, respectively. By Theorem 3.5, the lifting of any non-linear 4subclique of $\mathcal{C}$ is a centric cube of $\hat{\mathcal{X}}$ with center $n_{0}$. Hence, we can lift $\mathcal{C}$ to a subgraph of $\hat{\Gamma}$ which is the complement of figure 6 .

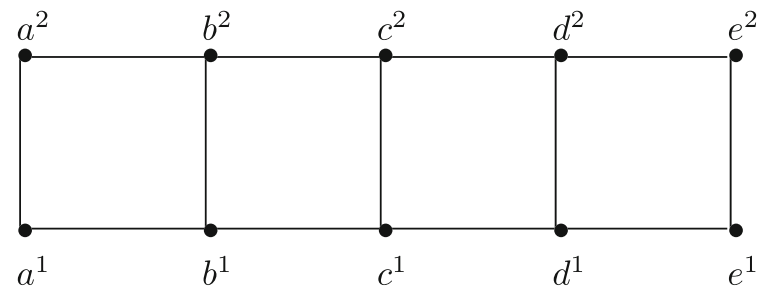

Figure 5. $(5 \times 2)$-grid as complement of a decade. 
Clearly, the complement of the graph in figure 6 is a dodecade. By definition of the canonical covering $\varphi_{0}$, each of the opposite pairs $\left\{a^{1}, a^{2}\right\},\left\{b^{1}, b^{2}\right\},\left\{c^{1}, c^{2}\right\},\left\{d^{1}, d^{2}\right\}$, $\left\{e^{1}, e^{2}\right\},\left\{f^{1}, f^{2}\right\}$ is on a secant line to $Q$ through $n_{0}$.

Every centric dodecade of $\hat{\Gamma}$ with center $n_{0}$ is mapped by $\varphi_{0}$ onto a non-linear 6-clique of $\Gamma$. This observation combined with Claim (b) of Proposition 3.10 proves Theorem 3(c).

The following theorem proves Theorem 2(a), (b).

\section{Theorem 3.11. The following hold:}

(a) The graph $\Gamma$ admits non-linear 5-cliques if and only if $q=2^{n}, n$ odd.

(b) If $q=2^{n}, n$ odd, then every 5-clique of $\Gamma$ is contained in a 6 -clique of $\Gamma$.

(c) The graph $\Gamma$ has no $k$-cliques with $k>6$.

Proof. Let $C:=\left\{a^{i}, b^{i}, c^{i}, d^{i}\right\}_{i=1,2}$ be the lifting of a non-linear 4-clique of $\Gamma$. Вy Theorem 3.5, $C$ is a centric cube of $\hat{\mathcal{X}}$. By the transitivity of the action of $S^{-}(6, q)$ on quadrangles of $Q$ and the proof of Lemma 3.6, without loss of generality, we can choose the cube $C_{\bar{p}}$ as in (9) of $\$ 3.3 .1$. In particular, $C_{\bar{p}}$ has center $\bar{p}=[u, 1 / u, v, 1 / v, r, s]$ with $u v \neq 0$ and $r^{2}+r s+\lambda s^{2}=1$.

We want to enlarge $C_{\bar{p}}$ to get a lifting of a $k$-clique of $\Gamma$ with $k \geq 5$.

Let $\bar{e}^{1}=\left[e_{1, i}\right]_{i=1}^{6}$ and $\bar{e}^{2}=\left[e_{2, i}\right]_{i=1}^{6}$ be two points of $Q$. The conditions on the coordinates of $\bar{e}^{i}(i=1,2)$ for the set $C_{\bar{p}} \cup\left\{\bar{e}^{i}\right\}_{i=1}^{2}$ to be a decade of $Q \backslash \bar{p}^{\perp}$ with center $\bar{p}$ are as follows (see Fig. 6):

$$
\begin{array}{lll}
\bar{b}^{1} \perp \bar{e}^{1}: & e_{1,4}=0 ; & \bar{d}^{1} \perp \bar{e}^{1}: e_{1,3}=0 ; \\
\bar{a}^{2} \perp \bar{e}^{1}: e_{1,1}=e_{1,5} u s+e_{1,6} u r ; & \bar{c}^{2} \perp \bar{e}^{1}: e_{1,2}=e_{1,5} s / u+e_{1,6} r / u ; \\
\bar{a}^{1} \perp \bar{e}^{2}: & e_{2,2}=0 ; & \bar{c}^{1} \perp \bar{e}^{2}: e_{2,1}=0 ; \\
\bar{b}^{2} \perp \bar{e}^{2}: & e_{2,3}=e_{2,5} s v+e_{2,6} r v ; & \bar{d}^{2} \perp \bar{e}^{2}: e_{2,4}=e_{2,5} s / v+e_{2,6} r / v .
\end{array}
$$

Since $\bar{e}^{-1}$ and $\bar{e}^{2}$ are points of $Q$, we have $f\left(\bar{e}^{1}\right)=0$ and $f\left(\bar{e}^{2}\right)=0$. Hence

$$
\begin{aligned}
& e_{1,5}^{2}\left(s^{2}+1\right)+e_{1,5} e_{1,6}+e_{1,6}^{2}\left(r^{2}+\lambda\right)=0, \\
& e_{2,5}^{2}\left(s^{2}+1\right)+e_{2,5} e_{2,6}+e_{2,6}^{2}\left(r^{2}+\lambda\right)=0,
\end{aligned}
$$

The points $\bar{e}^{-1}, \bar{e}^{2}$ and $\bar{p}$ must be on the same line. Hence

$$
e_{2,5}=e_{1,5}(1+r s)+e_{1,6} r^{2} \text { and } e_{2,6}=e_{1,5} s^{2}+e_{1,6}(1+r s) .
$$

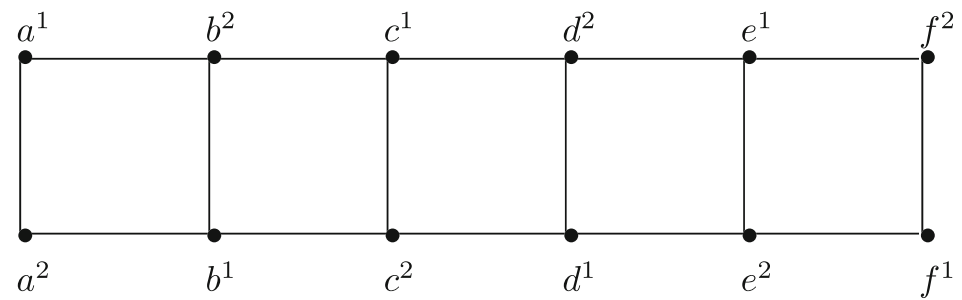

Figure 6. 6: $(6 \times 2)$-grid as complement of a dodecade. 
By plugging $e_{2,5}$ and $e_{2,6}$ of (17) in (16), we have

$$
e_{1,5}^{2}\left(\left(r^{2}+r s+\lambda s^{2}\right) s^{2}+1\right)+e_{1,5} e_{1,6}+e_{1,6}^{2}\left(r^{2}\left(r^{2}+r s+\lambda s^{2}\right)+\lambda\right)=0 .
$$

Since $r^{2}+r s+\lambda s^{2}=1$, the above equation gives us back (15). So, (16) is already implicit in (15). Hence we can disregard it.

The coordinates $e_{1, i}$ of $\bar{e}^{1}$ for $1 \leq i \leq 4$ and the coordinates $e_{2, i}$ of $\bar{e}^{2}$ for $1 \leq i \leq 4$ depend on $e_{1,5}$ and $e_{1,6}$. Hence we write $\bar{e}_{\left(e_{1,5}, e_{1,6}\right)}$ for $\bar{e}^{-1}$ and $\bar{e}_{\left(e_{1,5}, e_{1,6}\right)}^{2}$ for $\bar{e}^{2}$ to emphasize the dependance of $\bar{e}^{-1}$ and $\bar{e}^{2}$ on $e_{1,5}$ and $e_{1,6}$. Explicitly, we have

$$
\bar{e}_{\left(e_{1,5}, e_{1,6}\right)}=\left(e_{1,5} u s+e_{1,6} u r, e_{1,5} \frac{s}{u}+e_{1,6} \frac{r}{u}, 0,0, e_{1,5}, e_{1,6}\right)
$$

and

$$
\begin{aligned}
\bar{e}_{\left(e_{1,5}, e_{1,6}\right)}^{2}= & \left(0,0, e_{1,5} s v+e_{1,6} r v, \frac{s e_{1,5}}{v}+\frac{r e_{1,6}}{v},\right. \\
& \left.e_{1,5}(1+r s)+e_{1,6} r^{2}, e_{1,5} s^{2}+e_{1,6}(1+r s)\right) .
\end{aligned}
$$

Note that the possibility to enlarge $C_{\bar{p}}$ in order to get a lifting of a non-linear $k$-clique with $k \geq 5$ is equivalent to the existence of at least two points $\bar{e}^{1}$ and $\bar{e}^{2}$ as above. The number of choices for the pair $\left(\bar{e}^{1}, \bar{e}^{2}\right)$ determines the size of the $k$-clique. After having implemented all the collinearity relations among the points $C_{\bar{p}} \cup\left\{\bar{e}^{1}, \bar{e}^{2}\right\}$ to get a centric decade, we get that the points $\bar{e}^{-1}$ and $\bar{e}^{2}$ exist provided their coordinates satisfy (8) and (15). In the sequel, we will show that (8) and (15) are equivalent to $\operatorname{Tr}(1)=1$. Then, $\operatorname{Tr}(1)=1$ if and only if $q$ is an odd power of 2 (see e.g. [8]).

More precisely, we will deal with the cases $s=1$ and $s \neq 1$ separately showing that

(i) for $s=1$ and $s \neq 1$ the choices for $\left(\bar{e}^{1}, \bar{e}^{2}\right)$ allow exactly two extensions of $C_{\bar{p}}$ to decades;

(ii) the union of the two decades in (i) is a centric dodecade.

Suppose firstly $s=1$. Then (15) is

$$
e_{1,5} e_{1,6}+e_{1,6}^{2}\left(r^{2}+\lambda\right)=0
$$

and (8) becomes $\lambda=r^{2}+r+1$. So, $\operatorname{Tr}(\lambda)=\operatorname{Tr}\left(r^{2}+r+1\right)=\operatorname{Tr}(1)$. Since $\operatorname{Tr}(\lambda)=1$, we get

$$
\operatorname{Tr}(1)=1
$$

Suppose now $s \neq 1$. Then (15) is

$$
e_{1,5}^{2}+\frac{e_{1,5} e_{1,6}}{1+s^{2}}+\frac{\left(\lambda+r^{2}\right)\left(1+s^{2}\right) e_{1,6}^{2}}{\left(1+s^{2}\right)^{2}}=0 .
$$

Equation (22) admits solutions (in $e_{1,5}$ and $e_{1,6}$ ) if and only if

$$
\operatorname{Tr}\left(\lambda+\lambda s^{2}+r^{2}+r^{2} s^{2}\right)=0
$$

By (8) we have $\lambda s^{2}+r^{2}+r^{2} s^{2}=1+r s+r^{2} s^{2}$. Hence (23) becomes $\operatorname{Tr}\left(\lambda+\lambda s^{2}+r^{2}+\right.$ $\left.r^{2} s^{2}\right)=\operatorname{Tr}\left(\lambda+1+r s+r^{2} s^{2}\right)=0$ which implies that $\operatorname{Tr}(\lambda)+\operatorname{Tr}(1)+\operatorname{Tr}(r s)+\operatorname{Tr}\left(r^{2} s^{2}\right)=0$, i.e. $\operatorname{Tr}(1)=1$, again. 
Points $\bar{e}^{-1}$ and $\bar{e}^{2}$ exist if and only if (21) is satisfied, which is equivalent to require $q=2^{n}$ with $n$ odd. So, non-linear 5-cliques exist if and only if $q=2^{n}$ with $n$ odd. Claim (a) is proved.

Let $q=2^{n}$ and $n$ odd. Then (20) and (22) admit exactly two different solutions (in $e_{1,5}$ and $\left.e_{1,6}\right)$, up to proportionality: denote them by $\left(e_{1,5}^{\star}, e_{1,6}^{\star}\right)$ and $\left(e_{1,5}^{\circ}, e_{1,6}^{\circ}\right)$. According to (18) and (19) and keeping in mind that the exponents 1 and 2 refer to opposite points, put

$$
\bar{e}^{\star 1}:=\bar{e}_{\left(e_{1,5}^{\star}, e_{1,6}^{\star}\right)}^{1}, \bar{e}^{\star 2}:=\bar{e}_{\left(e_{1,5}^{\star}, e_{1,6}^{\star}\right)}, \bar{e}^{\circ 1}:=\bar{e}_{\left(e_{1,5}^{\circ}, e_{1,6}^{\circ}\right)}, \bar{e}^{\circ 2}:=\bar{e}_{\left(e_{1,5}^{\circ}, e_{1,6}^{\circ}\right)} .
$$

By construction, $C_{\bar{p}} \cup\left\{\bar{e}^{\star 1}, \bar{e}^{\star 2}\right\}$ and $C_{\bar{p}} \cup\left\{\bar{e}^{\circ 1}, \bar{e}^{\circ 2}\right\}$ are centric decades with center $\bar{p}$ and $C_{\bar{p}} \cup\left\{\bar{e}^{* 1}, \bar{e}^{* 2}, \bar{e}^{\circ 1}, \bar{e}^{\circ 2}\right\}$ is a centric dodecade with center $\bar{p}$. The theorem is proved.

The following is also implicit in the proof of Theorem 3.11 and corresponds to Theorem 2(d)(i) and (ii).

\section{COROLLARY 3.12}

Let $n$ be odd. Then every centric cube of $\hat{\mathcal{X}}$ is contained in exactly two centric decades and just one centric dodecade of $\hat{\mathcal{X}}$. Every centric decade of $\hat{\mathcal{X}}$ is contained in exactly one centric dodecade of $\hat{\mathcal{X}}$.

\section{PROPOSITION 3.13}

Let $\mathcal{N}_{i}, i \in\{2,3,4,5,6\}$, be the set of non-linear $i$-cliques in $\Gamma$ and $\left|\mathcal{N}_{i}\right|=N_{i}$. Then

(a) $N_{2}=q^{2}(q-1)\left(q^{4}-1\right) / 4 ; N_{3}=q^{4}(q-1)\left(q^{4}-1\right) / 12 ; N_{4}=q^{4}\left(q^{2}-1\right)\left(q^{4}-1\right) / 48$.

(b) Let $q=2^{n}>4$. If $n$ is even, then $\mathcal{N}_{5}=\mathcal{N}_{6}=\emptyset$. If $n$ is odd, then $N_{5}=q^{4}\left(q^{2}-1\right)$ $\left(q^{4}-1\right) / 120$ and $N_{6}=q^{4}\left(q^{2}-1\right)\left(q^{4}-1\right) / 720$.

Proof. Each non-linear $i$-clique, $i \in\{2,3,4,5\}$ in $\Gamma$ extends to a non-linear $(i+1)$ clique in $\Gamma$ in $q^{2}$ ways if $i=2$ (see calculation of $\lambda$ in $\$ 3.1$ ); in $q+1$ ways if $i=3$ (Theorem 3.9); in two ways if $n$ is odd and $i=4$; no way if $n$ even and $i=4$; and in a unique way if $n$ is odd and $i=5$ (Corollary 3.12). Any non-linear $(i+1)$-clique can be considered as an extension of any of its $i$-subcliques. Further, the number $N_{2}$ of 2-cliques (i.e., edges) in $\Gamma$ is $v k / 2$. So, $N_{3}=q^{2} N_{2} / 3, N_{4}=(q+1) N_{3} / 4, N_{5}=N_{6}=0$ if $n$ is even and, if $n$ is odd, $N_{5}=2 N_{4} / 5$ and $N_{6}=N_{5} / 6$. So, the proposition follows.

\subsection{Proof of Theorem 4}

(a) Let $H=\left\{a^{i}, b^{i}, c^{i}\right\}_{i=1,2}$ be a centric hexagon of $\hat{\mathcal{X}}$ with center $n_{0}$. The lines $\left\langle a^{1}, b^{1}\right\rangle$ and $\left\langle a^{2}, b^{2}\right\rangle$ are coplanar. So, they meet at a point $a^{0} \in H_{0} \cap Q$, where $H_{0}:=n_{0}^{\perp}$. Similarly, the lines $\left\langle b^{1}, c^{1}\right\rangle$ and $\left\langle b^{2}, c^{2}\right\rangle$ meet at a point $b^{0} \in H_{0} \cap Q$ and the lines $\left\langle a^{1}, c^{2}\right\rangle$ and $\left\langle a^{2}, c^{1}\right\rangle$ meet at the point $c^{0} \in H_{0} \cap Q$. Let $S:=\left\{a^{i}, b^{i}, c^{i},\right\}_{i=0,1,2}$ and consider the subgeometry $Q(S)$ of $Q$ induced on $S$ (see $\S 1.1$, paragraph before Theorem 4, for the definition), namely $Q(S):=\left(S, \mathcal{L}_{S}\right)$ where $\mathcal{L}_{S}=\{l \cap S: l$ is a line of Q such that $\mid l \cap$ $S \mid \geq 2\}$. 
It is straightforward to see that the subgeometry $Q(S)$ is an $\mathbb{F}_{2}$-subgeometry isomorphic to a classical $(2,1)$-generalized quadrangle $Q^{+}(3,2)$. By construction, the points $a^{0}, b^{0}, c^{0}$ are orthogonal to $n_{0}$ (with respect to the orthogonality relation $\perp$ defined by $Q)$. Hence $H \cong Q^{+}(3,2) \backslash n_{0}^{\perp}$.

(b) Let $C=\left\{a^{i}, b^{i}, c^{i}, d^{i}\right\}_{i=1,2}$ be a cube of $\hat{\mathcal{X}}$ with center $n_{0}$ and $\left\{\hat{l}_{1}, \hat{m}_{1}\right\}$, $\left\{\hat{l}_{2}, \hat{m}_{2}\right\}, \ldots,\left\{\hat{l}_{6}, \hat{m}_{6}\right\}$ be the six pairs of opposite edges of $C$. Then, for $j=1, \ldots, 6$, the lines $\hat{l}_{j}$ and $\hat{m}_{j}$ have the same point at infinity $l_{j}^{\infty}=m_{j}^{\infty}=: p_{j}$. These six points $p_{1}, p_{2} \ldots, p_{6}$ are partitioned into three pairs and each of these pairs is collinear in $Q_{0}$ with a given point $p_{0} \in Q_{0}$. Each of these three pairs of points (on a same line through $p_{0}$ ) corresponds to one of the three classes of parallel edges of the cube. The point $p_{0}$ is obtained as the sum of the 4 vertices of any face of the cube.

Let $S:=\left\{a^{i}, b^{i}, c^{i}, d^{i}\right\}_{i=1,2} \cup\left\{p_{i}\right\}_{0 \leq i \leq 6}$ and consider the subgeometry $Q(S)$ of $Q$ induced on $S$.

It is straightforward to see that $Q(S)$ is an $\mathbb{F}_{2}$-subgeometry isomorphic to $Q(4,2)$. It is also well known that the complement in $Q(4,2)$ of a tangent hyperplane is a cube. Hence, $C \cong Q(S) \backslash p_{0}^{\perp}$. Note that the projective subgeometry $\langle S\rangle_{\mathbb{F}_{2}}$ defined by $S$ over the field $\mathbb{F}_{2}$ contains the nucleus $n_{0}$ of $Q_{0}$ but $n_{0}$ is not the nucleus $n_{S}$ of the quadric $Q(S)$ (embedded in $\left.\langle S\rangle_{\mathbb{F}_{2}}\right)$.

(c) As remarked in $\S 1$, if $q=2$ then $\Gamma$ is a complete graph with 6 vertices. In this case, $Q \backslash Q_{0}$ is a dodecade.

Let $q \geq 4$. Since by assumption $q$ is an odd power of 2 , we can take $\lambda=1$ in (2); thus $Q$ is represented by the following equation:

$$
x_{1} x_{2}+x_{3} x_{4}+x_{5}^{2}+x_{5} x_{6}+x_{6}^{2}=0 .
$$

We shall count the number of subgeometries of $Q$ defined over the field $\mathbb{F}_{2}$ and represented by eq. (24) for a suitable choice of a basis. Equivalently, we count the number of different bases $B_{i}$ of the vector space $V=V(6, q)$ with the property that with respect to each of these bases $B_{i}$, the quadratic form induced by $f$ on the $\mathbb{F}_{2}$-space spanned by $B_{i}$ is a quadratic form of elliptic type over $\mathbb{F}_{2}$, hence equivalent to $x_{1} x_{2}+x_{3} x_{4}+x_{5}^{2}+$ $x_{5} x_{6}+x_{6}^{2}$.

Let us construct a basis $B=\left(b_{1}, b_{2}, b_{3}, b_{4}, b_{5}, b_{6}\right)$ as required above. We can choose $b_{1}, b_{2}, b_{3}, b_{4}$ so that $\left\langle b_{1}\right\rangle,\left\langle b_{2}\right\rangle,\left\langle b_{3}\right\rangle,\left\langle b_{4}\right\rangle$ is a quadrangle of $Q$. That is, $f\left(b_{i}\right)=0$ for $1 \leq i \leq 4, b_{1} \not \perp b_{3}, b_{2} \not \perp b_{4}$ and $b_{1} \perp b_{2} \perp b_{3} \perp b_{4} \perp b_{1}$.

The number of quadrangles of $Q$ is $\left(q^{3}+1\right)\left(q^{2}+1\right)(q+1) q^{6}$.

The condition $\alpha\left(b_{1}, b_{2}\right)=1$ and $\alpha\left(b_{3}, b_{4}\right)=1$ (where $\alpha$ stands for the bilinear form associated to $f$ ) still allows us to choose $b_{1}$ (or $\left.b_{2}\right)$ and $b_{3}$ (or $b_{4}$ ) up to arbitrary scalars. Hence, we have $\left(q^{3}+1\right)\left(q^{2}+1\right)(q+1) q^{6}(q-1)^{2}$ choices for the quadruple $b_{1}, b_{2}, b_{3}, b_{4}$. The vectors $b_{5}$ and $b_{6}$ are taken in the orthogonal space $\left\langle b_{1}, b_{2}, b_{3}, b_{4}\right\rangle^{\perp}$ of $\left\langle b_{1}, b_{2}, b_{3}, b_{4}\right\rangle$. (Recall that $\left\langle b_{1}, b_{2}, b_{3}, b_{4}\right\rangle^{\perp}$ is a projective line exterior to $Q$.) So, let us regard $b_{5}$ and $b_{6}$ as vectors of the 2 -dimensional vector space $\left\langle b_{1}, b_{2}, b_{3}, b_{4}\right\rangle^{\perp}$.

Let $f^{\prime}(x, y)=x^{2}+\mu x y+v y^{2}$ be the quadratic form induced by $f$ on $\left\langle b_{1}, b_{2}, b_{3}, b_{4}\right\rangle^{\perp}$, for a suitable choice of a basis $e_{1}, e_{2}$ of $\left\langle b_{1}, b_{2}, b_{3}, b_{4}\right\rangle^{\perp}$. Hence $\operatorname{Tr}\left(v / \mu^{2}\right)=1$ because the line $\left\langle b_{1}, b_{2}, b_{3}, b_{4}\right\rangle^{\perp}$ is external to $Q$. Put $b_{5}:=x_{1} e_{1}+x_{2} e_{2}$ and $b_{6}:=y_{1} e_{1}+y_{2} e_{2}$ for scalars $x_{1}, x_{2}, y_{1}, y_{2}$ to be determined in a few lines.

The conditions that are required on $b_{5}$ and $b_{6}$ are the following: $\alpha\left(b_{5}, b_{6}\right)=1, f\left(b_{5}\right)=$ 1 and $f\left(b_{6}\right)=1$. By Lemma 3.3, we have $q+1$ different choices for $b_{5}$. Indeed, $f\left(b_{5}\right)=$ 1 can be translated to $x_{1}^{2}+x_{1} x_{2}+x_{2}^{2}=1$.

Having chosen $b_{5}$, let us count how many choices remain for $b_{6}$. 
To fix ideas, put $b_{5}=e_{1}$. Then $\alpha\left(b_{5}, b_{6}\right)=1$ implies $y_{2}=1 / \mu$ and $f\left(b_{6}\right)=1$ implies $y_{1}^{2}+\mu y_{1} y_{2}+v y_{2}^{2}=1$, namely $y_{1}^{2}+y_{1}+v /\left(\mu^{2}\right)+1=0$. Since the equation $y_{1}^{2}+y_{1}+v /\left(\mu^{2}\right)+1=0$ has two distinct solutions, two different choices are left for $b_{6}$. A similar argument works with any other choice of $b_{5}$. Thus, in any case, only two choices are left for $b_{6}$ once $b_{5}$ has been chosen. So, the number of $\mathbb{F}_{2}$-subgeometries $Q(S)$ of $Q^{-}(5, q)$ isomorphic to $Q^{-}(5,2)$ is

$$
\begin{aligned}
\frac{\# \text { choices of }\left(b_{i}\right)_{i=1}^{6} \text { in } V(6, q)}{\# \text { choices of }\left(b_{i}\right)_{i=1}^{6} \text { in } V(6,2)} & =\frac{\left(q^{3}+1\right)\left(q^{2}+1\right)(q+1) q^{6}(q-1)^{2}(q+1) 2}{\left(2^{3}+1\right)\left(2^{2}+1\right)(2+1) 2^{6}(2-1)^{2}(2+1) 2} \\
& =\frac{\left(q^{3}+1\right)\left(q^{2}+1\right)(q+1)^{2} q^{6}(q-1)^{2}}{9 \cdot 5 \cdot 3^{2} \cdot 2^{6}} .
\end{aligned}
$$

By Proposition 3.13, the total number $\bar{N}_{6}$ of centric dodecades of $Q$ (any possible center being allowed) is

$(\sharp$ of dodecades with a given center $) \cdot|\mathrm{PG}(V) \backslash Q|$

$$
\begin{aligned}
& \left.=\mathrm{N}_{6} \cdot|\mathrm{PG}(V) \backslash Q|=\frac{\left(q^{4}-1\right)\left(q^{2}-1\right) q^{4}}{15 \cdot 48} \cdot\left[\frac{\left(q^{6}-1\right)}{q-1}-\left(q^{3}+1\right)(q+1)\right)\right] \\
& =\frac{\left(q^{3}+1\right)\left(q^{4}-1\right)\left(q^{2}-1\right) q^{6}}{48 \cdot 15} .
\end{aligned}
$$

Hence $\bar{N}_{6}=\frac{\left(q^{3}+1\right)\left(q^{4}-1\right)\left(q^{2}-1\right) q^{6}}{48 \cdot 15}$.

Note that if $Q(S)$ is an $\mathbb{F}_{2}$-subgeometry of $Q$ and $p \in\langle S\rangle_{\mathbb{F}_{2}} \backslash S$, then $Q(S) \backslash p^{\perp}$ is a dodecade with center $p$. For each such subgeometry $Q(S)$, there are $2^{2}\left(2^{3}+1\right)=4 \cdot 9$ possible choices for $p$. In view of (25), this accounts for a total of

$$
\frac{\left(q^{3}+1\right)\left(q^{2}+1\right)(q+1)^{2} q^{6}(q-1)^{2}}{9 \cdot 5 \cdot 3^{2} \cdot 2^{6}} \cdot 4 \cdot 9=\frac{\left(q^{3}+1\right)\left(q^{4}-1\right)\left(q^{2}-1\right) q^{6}}{5 \cdot 9 \cdot 2^{4}}=\bar{N}_{6}
$$

centric dodecades. Hence every dodecade with center $p$ corresponds to a subgeometry $Q(S) \backslash p^{\perp}$ over $\mathbb{F}_{2}$ as described above and vice versa.

\section{Acknowledgement}

The authors wish to thank Antonio Pasini for his very helpful remarks and comments on a first version of this paper.

\section{References}

[1] Bagchi B and Sastry N S N, One-step completely orthogonalizable codes from generalized quadrangles, Inf. Comput. 77 (1988) 123-130

[2] Brouns L, Thas J A and Van Maldeghem $\mathrm{H}$, A characterization of $Q(5, q)$ using one subquadrangle $Q(4, q)$, Europ. J. Comb. 23 (2002) 163-177

[3] De Bruyn B and Pasini A, Minimal underlying division rings of sets of points of a projective space, J. Algebra 318 (2007) 641-652

[4] De Clerck F and Van Maldeghem H, Some classes of rank 2 geometries, in: Chapter 10 of handbook of incidence geometry (ed.) F Buekenhout (1995) (Elsevier) 
[5] Debroey I and Thas J A, On semipartial geometries, J. Combin. Theory Ser. A 25(3) (1978) 242-250

[6] Hirshfeld J W P and Thas J A, General Galois geometries (1991) (Oxford: Clarendon Press)

[7] Hubaut $X$ and Metz R, A class of strongly regular graphs related to orthogonal groups (1983), Combinatorics '81 (Rome, 1981), Ann. Discrete Math., vol. 18 North-Holland, Amsterdam-New York, pp. 469-472

[8] Lidl R and Neiderreiter H, Finite fields, in: Encyclopedia of mathematics and its applications (1983) (Addison-Wesley Publishing Company), vol. 20

[9] Pasini A, Diagram geometries (1994) (Oxford: Oxford University Press)

[10] Payne S E and Thas J A, Finite generalized quadrangles, 2nd ed. (2009) (Zürich: EMS Series of Lectures in Mathematics)

[11] Pralle H, Affine generalized quadrangles- an axiomatization, Geometriae Dedicata 84 (2001) 1-23

[12] Thas J A and Payne S E, Spreads and ovoids in finite generalized quadrangles, Geometriae Dedicata 52 (1994) 227-253

Communicating Editor: Parameswaran Sankaran 